\title{
Recent advances in photo-crosslinkable hydrogels for biomedical applications
}

Jane Ru Choi ${ }^{\star, \ddagger, 1,2}$, Kar Wey Yong ${ }^{\star \star, \ddagger, 3}$, Jean Yu Choi ${ }^{4}$ \& Alistair C Cowie ${ }^{4}$

\section{ABSTRACT}

Photo-crosslinkable hydrogels have recently attracted significant scientific interest. Their properties can be manipulated in a spatiotemporal manner through exposure to light to achieve the desirable functionality for various biomedical applications. This review article discusses the recent advances of the most common photocrosslinkable hydrogels, including poly(ethylene glycol) diacrylate (PEGDA), gelatin methacryloyl (GelMA) and methacrylated hyaluronic acid (MeHA), for various biomedical applications. We first highlight the advantages of photopolymerization and discuss diverse photosensitive systems used for the synthesis of photo-crosslinkable hydrogels. We then introduce their synthesis methods and review their latest state of development in biomedical applications, including tissue engineering and regenerative medicine, drug delivery, cancer therapies and biosensing. Lastly, the existing challenges and future perspectives of engineering photocrosslinkable hydrogels for biomedical applications are briefly discussed.

\section{KEYWORDS:}

biosensing $\cdot$ cancer therapies $\cdot$ drug delivery - photo-crosslinkable hydrogels $\bullet$ tissue engineering

'Department of Mechanical Engineering, University of British Columbia, 2054-6250 Applied Science Lane, Vancouver, BC, V6T 1Z4, Canada; ${ }^{2}$ Centre for Blood Research, Life Sciences Centre, University of British Columbia, 2350 Health Sciences Mall, Vancouver, BC, V6T 1Z3, Canada; ${ }^{3}$ Department of Chemical\& Petroleum Engineering, Schulich School of Engineering, University of Calgary, Calgary, AB, T2N 7N4, Canada; ${ }^{4}$ Faculty of Medicine, University of Dundee, Dow Street, Dundee DD1 5EH, UK; *Author for correspondence: janeruchoi@gmail.com; **Author for correspondence: ronald_yong88@yahoo.com; ${ }^{\ddagger}$ Authors contributed equally

BioTechniques 66: 40-53 (January 2019)

10.2144/btn-2018-0083

\section{INTRODUCTION}

Hydrogels have been widely investigated as matrices for biomedical applications (e.g., tissue engineering and biosensing) owing to their crosslinking abilities under mild conditions, excellent biocompatibility, and tunable biochemical and biophysical properties [1-5]. As the structure and properties of hydrogels resemble the microenvironment of many human body tissues, they have been broadly used in a diverse range of biomedical applications $[6,7]$. To date, stimuli-responsive hydrogels have increasingly gained scientific interest. These materials can respond to various stimuli, including light, chemical and temperature to induce structural or morphological transitions $[8,9]$. Among various stimuli, light stimulus (e.g., ultraviolet [UV] light and visible light) is considered as an ideal external control method to manipulate the properties of hydrogels owing to their several unique advantages. These include the abilities to: (i) control the stimulus easily by switching the light on and off; (ii) regulate light dosage precisely to achieve functional tunability; (iii) obtain independent spatial or temporal control of different biological processes in a wavelength-specific manner; (iv) direct the light to a confined location at user-defined times; as well as (v) to regulate a range of cellular processes in vivo in the optical tissue window $[10,11]$. These advantages have led to the extensive use of light for better control and modulation of hydrogels for numerous potential applications.

Photo-crosslinkable hydrogels, including poly(ethylene glycol) diacrylate (PEGDA), gelatin methacryloyl (GelMA) and methacrylated hyaluronic acid (MeHA), have been extensively explored in biomedical fields, especially as 3D tissue engineered constructs, biosensing medium and drugcontrolled release matrices. This is due to their outstanding properties, such as good biocompatibility, hydrophilicity and biodegradability. For example, PEGDA hydrogel has been used in cartilage tissue engineering to mimic both static and dynamic mechanical properties of articular cartilage based upon their tunable mechanical properties [12]. GelMA hydrogel has been successfully used in the development of various 3D biomimetic in vitro healthy and disease tissue models for pathology and therapeutic studies [8]. MeHA hydrogels have been used in cancer therapy due to their abilities to target tumors for drug delivery applications [13]

The escalating demand for photocrosslinkable hydrogel as a platform for biomedical applications presents a strong need for a timely review on a wide range of their biomedical applications, including tissue engineering and regenerative medicine, biosensing, drug delivery and cancer therapies. This review discusses the latest advances of photo-crosslinkable hydrogels for biomedical applications. The three commonly used photo-crosslinkable hydrogels, including PEGDA, GelMA and MeHA, are highlighted. The advantages of using these hydrogels along with their different synthesis methods are summarized. Their most recent advances in biomedical applications, including studies published between 2013 and 2018, are subsequently reviewed (Table 1). Finally, the existing challenges and future perspectives of photo-crosslinkable hydrogels are briefly discussed.

\section{PHOTOSENSITIVE SYSTEM FOR THE SYNTHESIS OF PHOTO-CROSSLINKABLE HYDROGELS}

To date, a variety of photo-crosslinkable hydrogels have been developed with tunable mechanical and chemical properties (e.g., swelling rate, degradability and mechanical strength) for various biomedical applications [14]. As aforementioned, compared with other stimuli (e.g., $\mathrm{pH}$ or temperature), light stimulus is an interesting option as it can be easily controlled to precise locations at defined times and 


\section{BioTechniques}

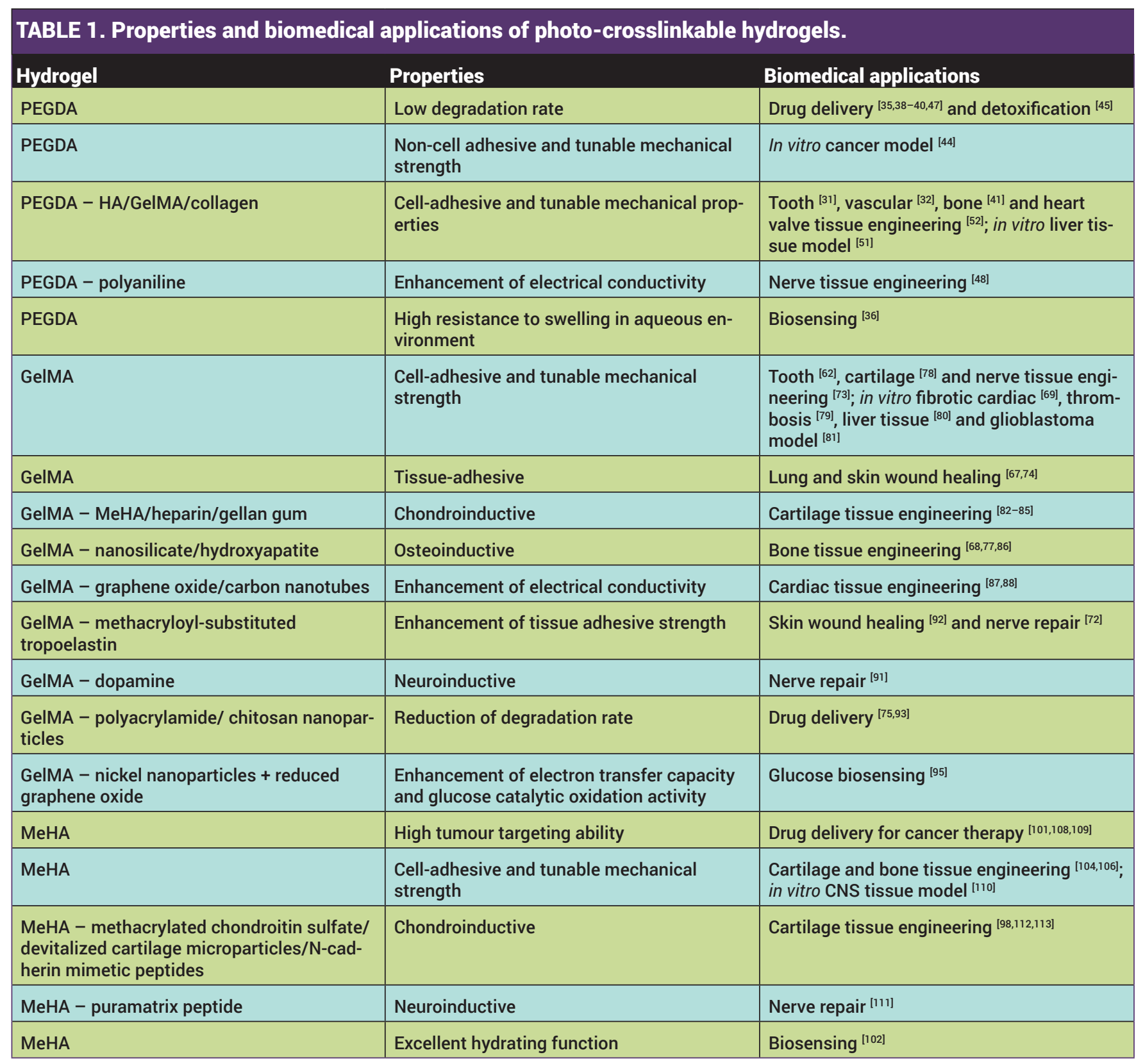

produce hydrogel structures with desirable properties [15]. A photosensitive system usually consists of photoinitiators, pre-polymers, and/or other components such as therapeutic molecules, cells and so on. The use of light energy (i.e., visible light [400-800 nm] or UV [200-400 nm]) enables polymerization of pre-polymer at low initiating radical doses under mild reaction conditions (i.e., in physiological aqueous solution at room temperature) [16]. The spatiotemporal control and resolution conferred by the light are essential to trigger the photopolymerization of pre-polymer in the presence of photoinitiator and induce the photosensitive bond cleavage to introduce specific biochemical functionalities to the hydrogel. The photopolymerization reaction and photoinitiators will be briefly discussed in the following sections.

\section{Photopolymerization reaction}

In general, photopolymerization reactions can be categorized into free-radicalinitiated chain polymerization and bio-orthogonal click reactions [17]. The former is the most common reaction used in the synthesis of hydrogels, which is normally based on methacrylate-functionalized pre-polymers and happens through a chain growth mechanism. The general processes of synthesizing three common photo-crosslinkable hydrogels, PEGDA, GelMA and MeHA, based on this reaction are shown in Figure 1. Briefly, in the presence of a light source, the photons absorbed by the photoinitiators promote their cleavage, leading to the formation of free radical molecules. These reactive molecules then react with the vinyl bonds in the pre-polymer (i.e., PEGDA, GelMA or $\mathrm{MeHA}$ ), leading to the formation of chemical crosslinks between the polymer chains [18]. This polymerization reaction allows the synthesis of hydrogels with tunable 
mechanical properties and degradation rate. However, radical-initiated chaingrowth polymerization possesses a few limitations. First, the inhibition effect of oxygen in the reaction has always been a key issue, as it leads to the production of peroxy radicals that can hardly contribute to the polymerization reaction $[17,19]$. This issue also causes a decrease in both polymerization rate and final conversion ratio, which results in the production of undesirable, highly viscous substances. Other limitations of radical-initiated chaingrowth polymerization include nonstructural heterogeneities in the polymer network, inefficient control of crosslinking polymerization kinetics, and the residual unreacted double bonds that might react negatively with biological molecules, which stem from the random chain polymerization [20].

Bio-orthogonal click reaction enables the development of hydrogels with consistent structure and minimal network defects based on the step-growth polymerization reaction [21]. Compared with chain-growth polymerization, this reaction is insensitive to oxygen and water, and is able to perform under mild conditions with faster kinetics, greater efficiency and selectivity [22]. The thio-norbornene (thiol-ene) photoclick reaction, in particular, has emerged as a widely used method for synthesis of hydrogel. In the presence of light irradiation, the thiol-ene reaction triggers the addition of thiols to carboncarbon double bonds in pre-polymers in a rapid manner, generating thioether bonds [23]. Like free-radical-initiated chain polymerization, this reaction enables the manipulation of mechanical properties and degradation rate of hydrogel $[22,24]$. Importantly, this reaction induces a low risk of cytotoxic effects to the cells, which makes it suitable for diverse biomedical applications, especially for tissue engineering applications [22]. To date, the practical applications of thiol-ene photoclick reaction remain challenging, as thiolcontaining small molecules have limited shelf life stability (several hours to several days) and they rely on stabilizers [25].

Photoinitiators for photopolymerization Selecting a suitable photoinitiator is imperative to ensure a desirable polymerization rate and to achieve optimum functionality for biomedical applications. For instance, using an appropriate photoinitiator could prevent undesirable cytotoxic effects for encapsulation of cells in tissue engineering applications [26]. Several key characteristics should be considered when selecting a photoinitiator, which include absorption spectrum, molar extinction coefficient, water solubility, stability and ability to produce free radicals $[23,26]$.

Generally, there are two main categories of photoinitiators that are currently used in the synthesis of hydrogels for biomedical applications: radical and cationic photoinitiators [26]. The former represents the most widely used photoinitiator owing to their excellent biocompatibility. By contrast, the utilization of cationic photoinitiators would lead to the formation of protonic acids that might be harmful to cells, which significantly restricts their applications [16]. In fact, radical photoinitiators are divided into two types: photo-cleavable (type I) and biomolecular (type II) photoinitiators, based on their mechanism in the production of free radicals. Type I photoinitiators such as benzoin derivatives and acetophenone derivatives have been commonly used [24]. Upon exposure to light, type I photoinitiators take up incident photons and decay into two primary radicals that initiate the photocrosslinking reaction. Type II photoinitiators, such as camphorquinone, thioxanthone and benzophenone, extract the hydrogen present in co-initiator to generate secondary radicals for the crosslinking process [23]. They have attracted considerable attention due to their utility in visible light-mediated polymerization.

When selecting the photoinitiators, the main concern is the potential cytotoxicity effect of free radicals produced by photoinitiators, especially for the application of tissue engineering [27]. Free radicals in turn can react with major cell components such as nucleic acids or proteins, which may reduce cell viability and even cause DNA damage. The commonly used photoinitiators for biomedical applications are summarized in Table 2. Irgacure 2959 (1-[4-(2-hydroxyethoxy)-phenyl]2-hydroxy-2-methyl-1-propane-1-one) is the most broadly used UV light-sensitive photoinitiator, especially in the field of tissue engineering, due to its fascinating characteristics such as moderate water solubility, low cytotoxicity and minimal immunogenicity [27]. However, its low initiation efficiency and low molar extinction coefficient in the UV-A spectral range have motivated the search for alternative photoinitiators that exhibit enhanced efficiency and biocompatibility such as 2,2'-azobis[2methyl-N-(2-hydroxyethyl)propionamide] (VA-086) and lithium phenyl-2,4,6-trimethylbenzoylphosphinate (LAP) [28]. Numerous efforts have been devoted to create the formulation of photosensitive systems that consists of visible-lightsensitive photoinitiators such as Eosin-Y, riboflavin and camphorquinone to alleviate possible harmful effects of UV irradiation on the encapsulated cells [23]. Compared with UV light-sensitive photoinitiators, visible light-sensitive photoinitiators are relatively less cytotoxic and highly watersoluble [29]. Their exceptional properties offer great promise for diverse applications. However, the key challenge is the rapidity of curing under visible light, as the energy level of the visible light is lower than that of UV light [16]. Therefore, more studies should be performed to improve the curing efficiency of pre-polymer under visible light. In short, selecting suitable photoinitiators is important to achieve desirable function of hydrogel for biomedical applications. The type and characteristics of each photoinitiator as well as the comparisons of both UV and visible photopolymerizations are summarized in Table 2.

\section{SYNTHESIS, PROPERTIES \& RECENT BIOMEDICAL APPLICATIONS OF PHOTO-CROSSLINKABLE HYDROGELS}

Photo-crosslinkable hydrogels basically consist of a photoreactive moiety that is usually made up of photochromic chromophore, and a polymeric network. The photochromic chromophore is photoresponsive upon light irradiation [3]. Generally, the optical signal is first captured by the photochromic chromophore, which in turn converts the optical signal to a chemical signal via a photoreaction, including isomerization, cleavage or dimerization [30]. The chemical signal is then transferred to the functional group of the hydrogel and attunes to its properties. The 
change in hydrogel properties may vary depending on their molecular structures and the photo-irradiation set-up [3]. The changes of hydrogel properties include elasticity, degree of swelling, and degradation, which are of interest for multiple biomedical applications. For instance, in the field of tissue engineering, the tunable properties of photo-crosslinkable hydrogels provide the dynamic and native cell microenvironment. These properties offer tremendous potential to study the unknown mechanisms of the complex cell microenvironment [31-33]. For drug delivery, photo-crosslinkable hydrogels offer the opportunity for on-demand delivery of different therapeutics (e.g., drugs and growth factors) in a single cytocompatible hydrogel-based drugdelivery system with high loading efficiency. The hydrogel could achieve dose-controlled and timed release of drugs at desired sites for therapeutic applications [34].

\section{PEGDA hydrogel}

PEG is a hydrophilic, water soluble and biocompatible polymer, which has been suggested for use in various biomedical applications [35]. The polymer is nontoxic at molecular weighs above $400 \mathrm{Da}$, has low immunogenicity, and is readily excreted from the human body. It has been approved by the US FDA for internal consumption. The PEG molecule is neutral, highly mobile and hydrated in aqueous solution [36]. It is naturally resistant to protein adsorption due to its limited protein binding sites and high mobility, and hence it does not support cell attachment [37]. PEGDA is a PEG derivative fabricated through substituting terminal hydroxyl groups of PEG with acrylates. It contains double-bond acrylate groups at each end of the PEG chain, allowing it to undergo free radical initiated chain photopolymerization in the presence of a photoinitiator molecule (e.g., Irgacure 2959) to form a 3D polymer network.

While PEGDA can be crosslinked by various methods, the utilization of photopolymerization is especially versatile for use in biomedical applications. In this regard, crosslinking can be induced through the addition of biocompatible photoinitiators in the presence of UV or visible light source [38]. PEGDA hydrogels

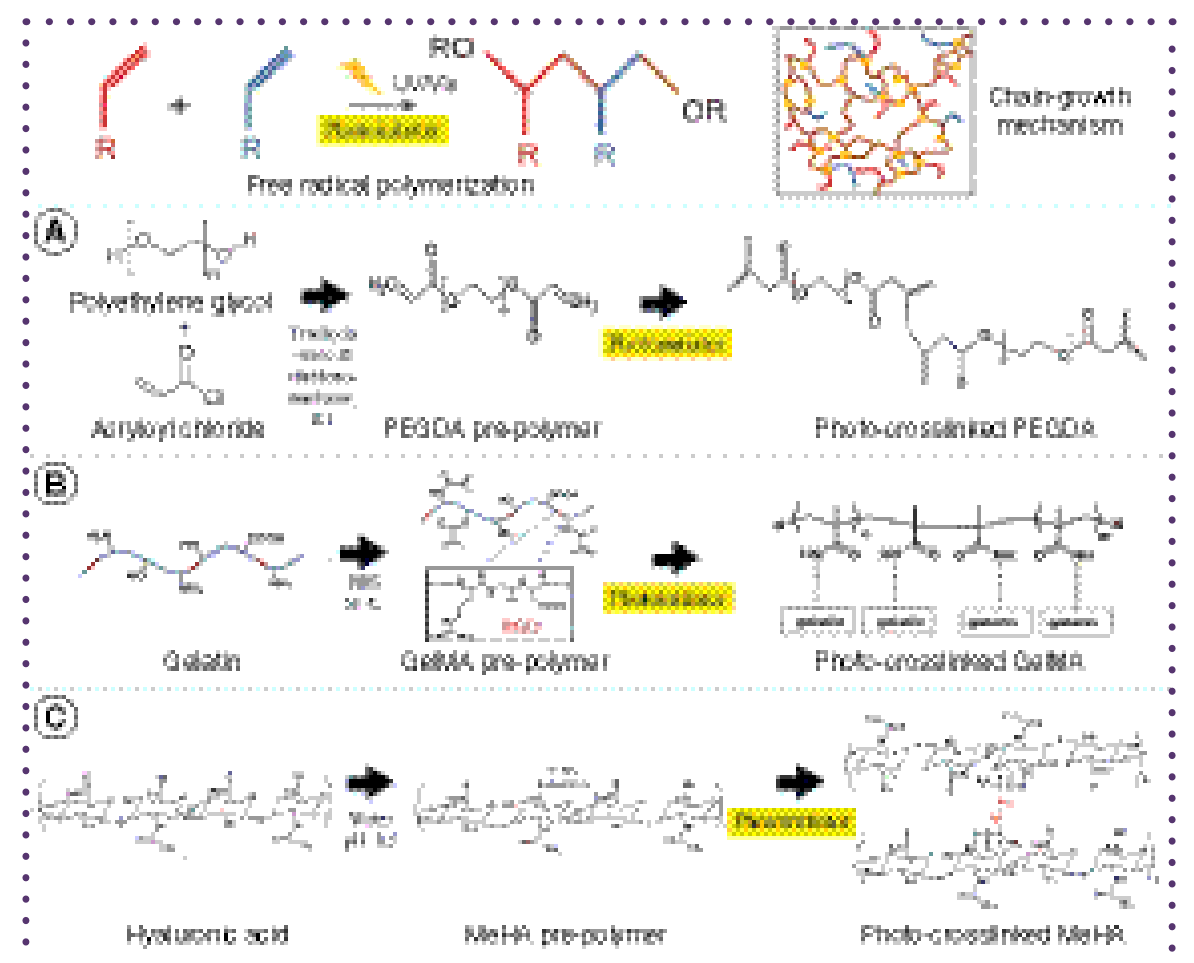

Figure 1. Photopolymerization of PEGDA (A), GeIMA (B) and MeHA (C) hydrogels via free-radical initiated chain polymerization. These images were adapted with permission from [109] $\odot$ Royal Society of Chemistry (2016) and [16].

GeIMA: Gelatin methacryloyl; MeHA: Methacrylated hyaluronic acid; PEGDA: Poly(ethylene glycol) diacrylate.

can be synthesized rapidly and easily, and their mechanical properties can be readily tuned by varying the concentration or molecular weight (MW) of the polymer [39]. These properties make it a promising biomaterial for various biomedical applications, including drug delivery, tissue engineering and biosensing [40-42].

\section{Synthesis of photo-crosslinkable PEGDA hydrogel}

To synthesize PEGDA, PEG diol is first dissolved in benzene, followed by the removal of water through azeotropic distillation in toluene using a Dean-Stark trap [43]. This is to ensure that the acrylation of PEG is carried out in a dry condition. The PEG acrylation is then carried out under argon by dissolving PEG in dichrolomethane solution with acryloyl chloride and triethylamine at a molar ratio of 1:1.5:1.5 of OH-groups of PEG: acryloyl chloride: triethylamine, respectively. The mixture is stirred in the dark at room temperature overnight. The product is then precipitated by the addition of diethyl ether and chilled to $4^{\circ} \mathrm{C}$ to eliminate toluene and subsequently recovered using filtration. Finally, the recovered PEGDA product is dried in a vacuum oven overnight.

To synthesize PEGDA-based hydrogel, the PEGDA powder is dissolved in deionized water at a predetermined mass proportion (e.g., 0.25 g PEGDA/ml deionized water) [38]. Photoinitiator Irgacure 2959 is usually added to the PEGDA solution at $0.1 \%(\mathrm{w} / \mathrm{v})$ and is subsequently exposed to UV light for a few minutes (e.g., 10-20 min) to complete photopolymerization. The mechanical properties of PEGDA hydrogel are tunable by varying MW cut-offs (MWCOs), which generally lie in the $1-50 \mathrm{kDa}$ range; pre-polymer concentration; photopolymerization conditions, and so on. For example, by increasing the pre-polymer concentration from 5 to $25 \%$, compressive modulus of PEGDA hydrogel was increased from 5 to $70 \mathrm{kPa}$ [44] (Table 3). On the other hand, when the pre-polymer concentration was increased from 5 to $10 \%$, hydrolytic degradation of PEGDA hydrogel was decreased from 20 to $10 \%$ after 28 days of 


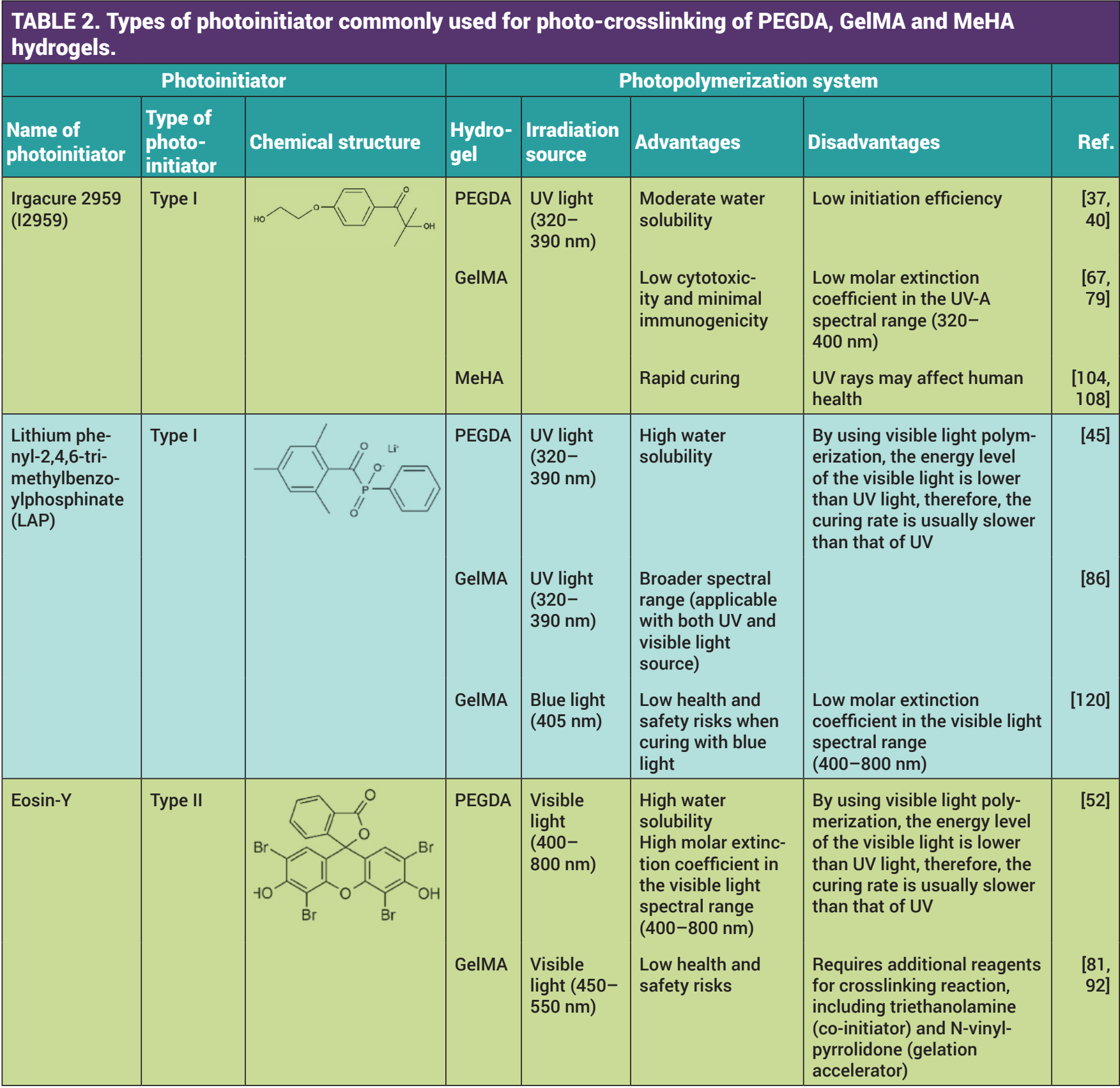

- incubation in phosphate-buffered saline (PBS). Due to its low degradation rate, PEGDA hydrogel acts as a stable medium to protect encapsulated drugs or functional nanoparticles for long-term drug release or detoxification, respectively $[39,45]$. However, in order to use PEGDA hydrogel for tissue engineering applications, the degradation rate of PEGDA hydrogel should be increased to allow cells to remodel their microenvironment or extracellular matrix [46]. The degradation rate of PEGDA hydrogel can be improved by combining it with other enzymatically or hydrolytically degradable materials [32]. For instance, degradation of PEGDA was increased up to $80 \%$ after 14 days of immersion in collagenase solution in which GelMA was added to form PEGDA/GelMA hybrid hydrogel for tooth tissue engineering [31].

RECENT BIOMEDICAL APPLICATIONS OF PHOTO-CROSSLINKABLE PEGDA HYDROGEL Drug delivery

Photo-crosslinkable PEGDA hydrogel is primarily used in drug delivery for therapeutic applications. UV-crosslinked PEGDA hydrogel was made into pharmaceutical tablets using 3D inkjet printing to control the release of ropinorole hydrochloride, a drug used for the treatment of Parkinson's disease [38]. It has also been made into microneedles using a micromolding approach to deliver gap junction inhibitor (Gap 26) for repair of keloid scars via the transdermal route [40] (Figure 2A). In addition, PEGDA hydrogel can be used as an implant to control a sustained release of stromal cell-derived factor 1/glycoprotein $\mathrm{VI}$, which promotes migration and engraftment of bone marrow-derived stem 


\begin{tabular}{|c|c|c|c|c|c|}
\hline Hydrogel & $\begin{array}{l}\text { Pre-polymer } \\
\text { concentration } \\
(w / v)\end{array}$ & $\begin{array}{l}\text { UV light exposure } \\
\text { duration }\end{array}$ & $\begin{array}{l}\text { Photoinitiator } \\
\text { concentration } \\
(\mathrm{w} / \mathrm{v})\end{array}$ & $\begin{array}{l}\text { Mechanical } \\
\text { properties }\end{array}$ & Biomedical application \\
\hline PEGDA & $5-25 \%$ & $5 \mathrm{~min}$ & $1 \%$ & $\begin{array}{l}\text { Compressive } \\
\text { modulus (2- } \\
70 \mathrm{kPa})\end{array}$ & $\begin{array}{l}\text { PEGDA hydrogel with different } \\
\text { compressive modulus can be used } \\
\text { to develop various types of } 3 \mathrm{D} \text { in } \\
\text { vitro cancer models, such as breast } \\
\text { cancer }(5 \mathrm{kPa}) \text {, gastrointestinal } \\
\text { cancer }(25 \mathrm{kPa}) \text {, and bone cancer } \\
(50 \mathrm{kPa})^{[44]} \text {. }\end{array}$ \\
\hline GelMA & $10-25 \%$ & $3 \mathrm{~min}$ & $0.5 \%$ & $\begin{array}{l}\text { Adhesive } \\
\text { strength (5- } \\
49 \mathrm{kPa}) \text { Elastic } \\
\text { modulus (175- } \\
262 \mathrm{kPa}) \\
\end{array}$ & $\begin{array}{l}25 \% \text { GelMA hydrogel is an excel- } \\
\text { lent sealant of air leakage for highly } \\
\text { stressed elastic lung tissues due to } \\
\text { its great adhesive strength }(49 \mathrm{kPa}) \\
\text { and elastic modulus }(262 \mathrm{kPa})^{[67]} \text {. }\end{array}$ \\
\hline GelMA & $5-15 \%$ & $30 \mathrm{~s}$ & $0.1 \%$ & $\begin{array}{l}\text { Elastic modulus } \\
(1.5-12 \mathrm{kPa})\end{array}$ & $\begin{array}{l}15 \% \text { GelMA hydrogel with an elas- } \\
\text { tic modulus of } 12 \mathrm{kPa} \text { supported } \\
\text { growth of odontoblast for engineer- } \\
\text { ing of dental pulp tissue construct } \\
\text { for tooth regeneration }{ }^{[62]} \text {. }\end{array}$ \\
\hline GelMA & $3 \%$ & $15-40 \mathrm{~s}$ & $0.5 \%$ & $\begin{array}{l}\text { Compressive } \\
\text { modulus }(0.68- \\
2.03 \mathrm{kPa})\end{array}$ & $\begin{array}{l}\text { UV light exposure for } 15 \text { s produced } \\
\text { a more softer GelMA hydrogel } \\
(0.68 \mathrm{kPa}) \text {, which is similar to that of } \\
\text { native brain tissue, allowing neuro- } \\
\text { nal differentiation of stem cells en- } \\
\text { capsulated in the hydrogel for spinal } \\
\text { cord repair }{ }^{[73]} \text {. }\end{array}$ \\
\hline GelMA & $7 \%$ & $2-10 \mathrm{~min}$ & $10 \%$ & $\begin{array}{l}\text { Young's modulus } \\
(290-350 \mathrm{kPa}) \\
\text { Tensile strength } \\
(310-360 \mathrm{kPa})\end{array}$ & $\begin{array}{l}\text { UV light irradiation for } 10 \text { min gen- } \\
\text { erated a GelMA hydrogel with high } \\
\text { elasticity }(350 \mathrm{kPa}) \text { and tensile } \\
\text { strength }(360 \mathrm{kPa}) \text {, which is com- } \\
\text { parable with that of natural skin, for } \\
\text { efficient wound healing }{ }^{[74]} \text {. }\end{array}$ \\
\hline MeHA & $1-3 \%$ & $10 \mathrm{~min}$ & $0.1 \%$ & $\begin{array}{l}\text { Elastic modulus } \\
(1.3-10.6 \mathrm{kPa})\end{array}$ & $\begin{array}{l}2.5 \% \text { MeHA hydrogel with an elastic } \\
\text { modulus of } 6.8 \mathrm{kPa} \text { is the optimal } \\
\text { gel formulation for } 3 \mathrm{D} \text { bioprinting } \\
\text { and bone tissue engineering }{ }^{[106]} \text {. }\end{array}$ \\
\hline
\end{tabular}

cells at the ischemic region of cardiovascular tissues for angiogenesis and cardiac regeneration [35]. Due to the non-cell adhesive property of PEGDA hydrogel, it was used to encapsulate islets of Langerhans and maintain their round morphology, preserving their insulinsecreting ability. By combining with a vascularized hydrogel, this construct can be implanted for the treatment of diabetes [37]. To deliver the drug in harsh conditions in the human body (e.g., highly impermeable endothelium and extremely low $\mathrm{pH}$ ), PEGDA hydrogel requires combination with other materials. For instance, a stent coated with swellable PEGDA/ polylactic acid-co-caprolactone hybrid hydrogel was developed to deliver the drug mitomycin $\mathrm{C}$ through the highly impermeable barrier of urothelium for treating diseases. This hybrid hydrogel firmly adheres to the urothelial mucosa and swells upon urine absorption, which enhances bioavailability of the drug at the urothelial diseased site [39]. PEGDA/ chitosan-grafted-glycidyl methacrylate hybrid hydrogel functionalized with bone ash has been used to efficiently control the release of antibiotic amoxicillin in an extremely low $\mathrm{pH}$ condition ( $\mathrm{pH}: 1.2, \mathrm{pH}$ of the gastric environment), suggesting great potential as a drug carrier for treatment of gastric diseases [47].

\section{Tissue engineering}

In addition to drug delivery, PEGDA hydrogel has also been used for tissue engineering. The pristine PEGDA lacks cell adhesive properties. Due to this, it allows growth of cancer cells and formation of tumorspheres, indicating its great potential in developing various types of $3 \mathrm{D}$ in vitro cancer model, such as a breast cancer model, gastrointestinal cancer model and bone cancer model [44]. However, in order to utilize it as a 3D cell culture platform for developing tissueengineered constructs or implants, it has to be combined with other biomaterials possessing cell adhesive properties, such as HA, GelMA, collagen and polyaniline $[31,32,41,48]$. PEGDA/GelMA hybrid hydrogel and PEGDA/collagen hybrid hydrogel were loaded with adult stem cells, respectively, for tooth and vascular regeneration [31,32]. PEGDA/HA hybrid hydrogel was employed to promote bone regeneration, which can be functionalized with laponite XLG nanoclay (a composite that releases bioactive ions $\mathrm{Mg}^{2+}$ and 
$\checkmark \mathrm{Si}^{4+}$ ) to create a favorable microenvironment for in vivo osteogenesis [41] (Figure 2B). Besides providing matrix for cell adhesion, polyaniline also enhances electrical conductivity of PEGDA hydrogel and neuronal differentiation, making this hybrid hydrogel suitable for regeneration of nerve cells [48]. Moreover, a low-level laser treatment can be conducted on a cell-laden PEGDA-based hydrogel to promote neuronal differentiation for neural tissue regeneration. For example, a red laser light $(633 \mathrm{~nm})$ with a low power output $(750 \mathrm{mw})$ has been used to induce neuronal differentiation and suppress glial differentiation of neural stem cells encapsulated in UV light-crosslinked PEGDA/GelMA hybrid hydrogel [49]. On the other hand, it is suggested that uniform-sized embryoid bodies (EBs) derived from embryonic stem cells should be formed in order to efficiently differentiate EBs into neuronal cells. To this end, a 3D PEGDA microwell was developed to enhance aggregation of embryonic stem cells due to its cell-repellent property, thus promoting formation of uniformsized EBs. Following encapsulation of such EBs in GelMA hydrogel, neurites were found to extend into both hydrogels to form neuronal networks. This construct can be used to develop various in vitro neural disease models, such as Alzheimer's disease, for exploring new and effective therapies [50]. Aside from UV light-crosslinked PEGDA hydrogel, visible light-crosslinked PEGDA hydrogel functionalized with RGD peptides can also be used to develop in vitro liver tissue models and tissue engineered heart valves $[51,52]$.

\section{Biosensing}

PEGDA hydrogels have been used in the biosensing or fabrication of microfluidic devices due to their high resistance to swelling in an aqueous environment, thus reducing sample loss [36]. For instance, one study has introduced PEG-based microfluidic chips integrated with functionalized nanoporous alumina membrane for simultaneous electrochemical detection of Escherichia coli 0157:H7 and Staphylococcus aureus [36]. In another study, PEGDA hydrogel was made into microparticles and conjugated with a specific antibody for the detection of clinically relevant markers (e.g., VEGF) based on silver precipitation, achieving a rapid and highly sensitive assay for pointof-care testing [53]. PEGDA have also been used in microfluidic devices as a barrier between the cell culture chambers and sensing chambers [42] (Figure 2C). Growth factors produced by hepatocytes diffuse into the sensing chamber through the hydrogel barrier, which were bound to the beads coated with fluorescence-tagged antibody. The fluorescence signal was then detected to determine the concentration of growth factors.

\section{Others}

Besides mammalian cell culture, PEGDA hydrogel may be used as a barrier in a microbial cell culture platform for nutrient and oxygen transport to microbes, owing to its tunable mesh size [54]. Polydiacetylene nanoparticle-laden PEGDA hydrogel with liver-mimetic 3D microstructure has been used as a detoxification device to effectively capture and remove toxins from the human body [45] (Figure 2D).

\section{GellMA hydrogel}

Gelatin is a FDA-approved material for food processing. It is an attractive base material of hydrogels due to its biodegradability, biocompatibility and low antigenicity $[55,56]$. Gelatin is a hydrolysis product of collagen that contains many arginine-glycine-aspartic acid (RGD) sequences and target sequences of matrix metalloproteinase (MMP) that enhance cell attachment and remodeling of the cellular microenvironment, respectively $[8,57]$. However, gelatin hydrogel exhibits a relatively rapid degradation behavior and poor mechanical strength [58], restricting its biomedical applications. GelMA hydrogel, a gelatinbased hydrogel functionalized with methacrylic anhydride (MA), was first introduced in the year 2000 [59]. The methacrylation process has been proven to control degradation rate and improve the mechanical strength of gelatin $[60,61]$. This makes GelMA hydrogel suitable for various biomedical applications, including tissue engineering and regenerative medicine, drug, growth factor and gene delivery [62-64].
Synthesis of photo-crosslinkable GelMA hydrogel

To synthesize GelMA in the conventional manner, the first step is to methacrylate gelatin (substitution of methacryloyl groups on the hydroxyl and reactive amine groups of gelatin) in PBS ( $\mathrm{pH}=7.4)$ at $50^{\circ} \mathrm{C}$, producing the GelMA pre-polymer solution [59]. The next step is to terminate the methacrylation reaction by adding phosphate buffer solution followed by dialysis against deionized water using a dialysis tubing with $\mathrm{MW}$ cut-offs that range from 12 to $14 \mathrm{kDa}$ for 5 to 7 days. The purpose of this step is to remove all the impurities with low MW (e.g., MA byproducts and unreacted MA) that are potentially cytotoxic. Finally, GelMA pre-polymers are produced by freeze-drying the dialyzed solution [8]. However, the conventional method requires MA concentrations more than tenfold higher than that of gelatin to produce GelMA with a high degree of methacrylation (>85\%). This is because PBS is insufficient to maintain the $\mathrm{pH}$ of the reaction solution at neutral for methacrylation to take place efficiently due to the presence of a byproduct, methacrylic acid, which causes the solution to become acidic. Therefore, a novel and efficient GelMA synthesis method that involves the use of carbonate-bicarbonate (CB) buffer has been developed. Gelatin can be methacrylated in a $\mathrm{CB}$ buffer at a $\mathrm{pH}$ of 9 at $35-50^{\circ} \mathrm{C}$ to successfully reduce the concentration ratio of MA to gelatin down to 2.2-fold and generate GelMA with a higher degree of methacrylation (96\%) compared with the conventional synthesis method [65].

GelMA pre-polymers (1 to $15 \%$ ) (w/v) form GelMA hydrogel constructs in the presence of an energy source (UV or visible light) and a photoinitiator using various microfabrication strategies (e.g., photopatterning, micromolding and bioprinting) $[8,55]$. By increasing the pre-polymer concentration, elastic modulus and adhesive strength of GelMA hydrogel can be tuned [66,67]. For instance, when the pre-polymer concentration was increased from 10 to $25 \%$, elastic modulus of GelMA hydrogel was found to increase from 175 to $262 \mathrm{kPa}$, while the adhesive strength was increased from 5 to $49 \mathrm{kPa}$ [67] (Table 3). Moreover, 
by adjusting the amount of MA, different degrees of methacrylation can be achieved, generating GelMA hydrogels with different mechanical properties. It has been reported that increasing the degrees of methacrylation enhanced the compressive modulus of GelMA hydrogel and reduced its degradation rate $[66,68,69]$. Photo-crosslinking of GelMA via radical polymerization is usually performed using Irgacure 2959 in the presence of UV light $[66,70]$. Irgacure 2959, a water-soluble photoinitiator is usually added into GelMA, and dissociates into a ketyl- and benzoyl-free radical, which has a low risk of cytotoxicity upon UV light exposure [71]. Photoinitiator concentration and light exposure duration are other parameters that can be used to tune the mechanical properties of GelMA hydrogel [59]. For example, by increasing concentration of Irgacure 2959 from 0.1 to $0.5 \%(\mathrm{w} / \mathrm{v})$, elastic modulus of GelMA hydrogel was increased from 7 to $10 \mathrm{kPa}[62,72]$. On the other hand, compressive modulus of GelMA hydrogel was increased from 0.68 to $2.03 \mathrm{kPa}$ when UV light exposure duration was increased from 15 to $40 \mathrm{~s}$ (Table 3) [73]

Besides mechanical properties, degradation rate of GelMA hydrogel can be tuned by varying the pre-polymer concentration and light exposure duration [62,67,74]. For instance, when the pre-polymer concentration was increased from 5 to 15\%, enzymatic degradation of GelMA hydrogel was decreased from 83 to $22 \%$ after 1 day of incubation in collagenase solution [62]. On the other hand, when UV light exposure duration was increased from 2 to $10 \mathrm{~min}$, degradation of GelMA hydrogel was decreased from 90 to $60 \%$ after 28 days of immersion in collagenase solution [74]. Moreover, some organic and inorganic materials such as chitosan nanoparticle, 4-arm PEG-tetraacrylate (PEGTA), methacryloyl-substituted tropoelastin and nickel have been shown to reduce the degradation rate of GelMA hydrogel [72,75-77]. In fact, the degradation rate of the GelMA-based hydrogel construct should match with the pace of neo-tissue formation for tissue engineering applications, or the drugrelease kinetics for drug-delivery applications $[72,75]$.
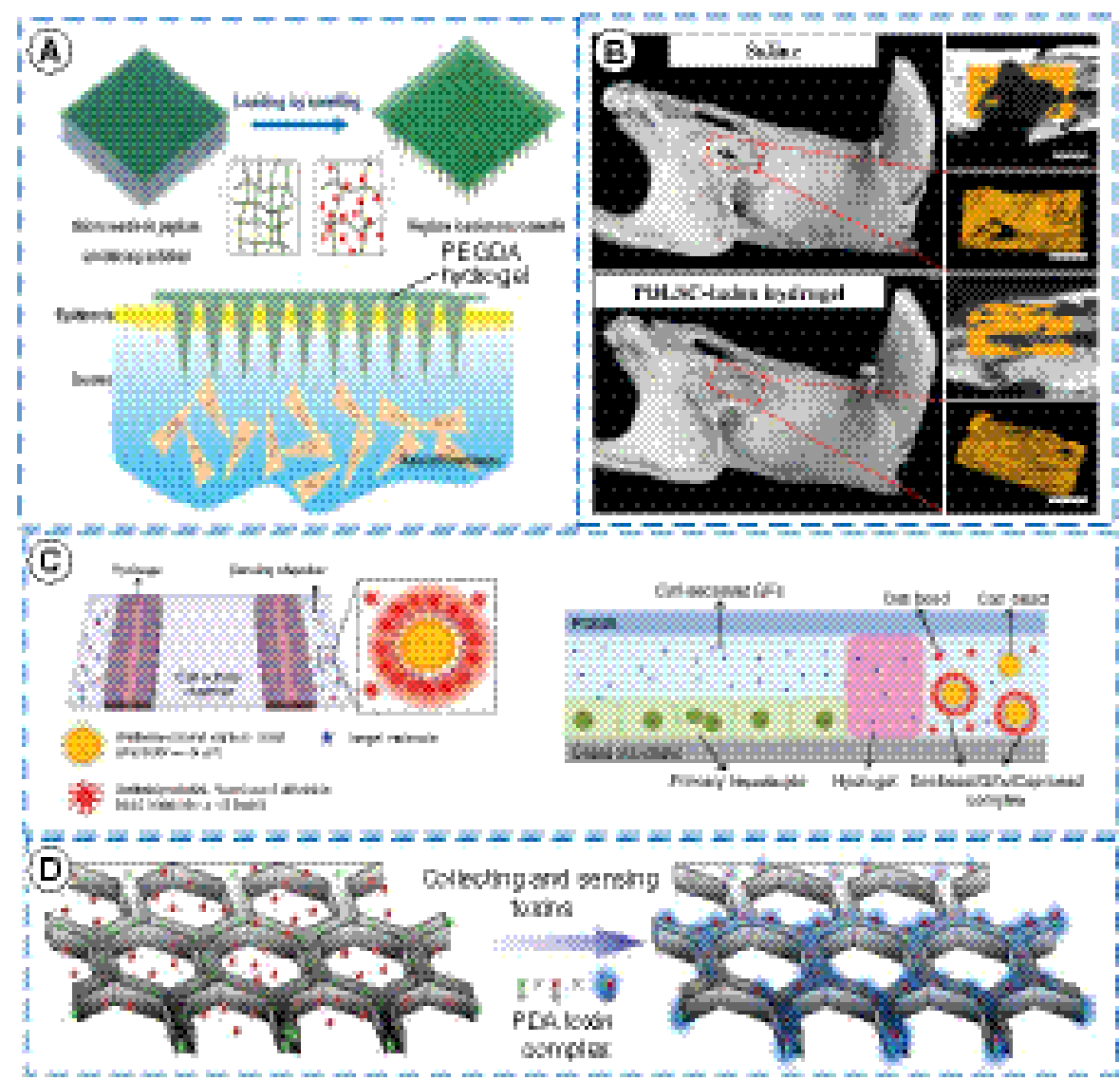

Figure 2. Biomedical applications of PEGDA hydrogel. (A) Microneedle-based drug delivery for treatment of skin disease; adapted with permission from [40]. (B) Bone regeneration; adapted with permission from [41]. (C) Sensing of cell-secreted GFs; adapted with permission from [42]. (D) Detoxification; adapted with permission from [45].

Cap: Capture; Det: Detector; GF: Growth factor; PDA: Polydiacetylene; PDLSC: Periodontal ligament stem cell; PEGDA: Poly(ethylene glycol) diacrylate.

\section{RECENT BIOMEDICAL APPLI- CATIONS OF PHOTO-CROSS- LINKABLE GELMA HYDROGEL}

Tissue engineering

Photo-crosslinkable GelMA hydrogel is primarily used for tissue engineering and regenerative medicine. UV light-crosslinked GelMA hydrogel has been used to create tissue engineered cartilage- and pre-vascularized dental pulp-like constructs for cartilage and tooth regeneration (Figure 3A), respectively $[62,78]$. Bioprinted articular cartilage-resident chondroprogenitor cellladen GelMA hydrogel construct was shown to be more effective in nonhypertrophic neo-cartilage formation than chondrocyteor mesenchymal stem cell-laden GelMA hydrogel construct [78]. In addition, GelMA hydrogel has been used for the engineering of $3 \mathrm{D}$ in vitro fibrotic cardiac and blood coagulation tissue models to study the fibrotic remodeling process in the heart, and thrombosis, respectively [69,79]. It has also been used to develop a liver-on-a-chip platform for drug toxicity assessments (e.g., acetaminophen toxicity) [80]. Moreover, transplantation of neural stem cells encapsulated in GelMA hydrogel was found to enhance neuronal regeneration and suppress glial scar formation in mouse spinal cord transection models, suggesting the potential therapeutic application of the construct in spinal cord injury [73]. Apart from UV light-crosslinked GelMA hydrogel, visible light-crosslinked GelMA hydrogel has been used to engineer $3 D$ in vitro glioblastoma multiform tissue models for monitoring cancer progression and drug screening [81]. On the other hand, implantation of a cell-free UV light-crosslinked GelMA hydrogel into animal models of lung injury was found to be effective at sealing 
air leak (Figure 3B) and promote in vivo wound healing for skin regeneration $[67,74]$. GelMA sealant appears to be more suitable for clinical applications as it displays excellent adhesive properties compared with the commonly used PEG- and fibrin-based glues [67].

Using GelMA hydrogel as a sole material may be suboptimal for biomedical applications. Therefore, incorporating other inorganic or organic components into GelMA is important to further improve its mechanical and chemical properties, as well as cell behavior (e.g., cell differentiation, proliferation and viability) [8]. For example, it was found that chondrocyte-laden pristine GelMA hydrogel shows deposition of extracellular matrix consisting of mainly collagen type I instead of collagen type II, indicating a poor quality of cartilage tissue construct [82]. By adding MeHA, heparin or gellan gum into the GelMA hydrogel construct, chondrocytes encapsulated in such 3D hybrid hydrogels maintain their chondrogenic phenotypes and increase the deposition of collagen type II [82-85]. For bone tissue engineering applications, GelMA hydrogel loaded with nanosilicate or hydroxyapatite was shown to enhance osteogenic differentiation of mesenchymal stem cells and bone mineralization in the absence of any osteoinducting factors $[77,86]$. Moreover, by incorporating a bioprinted vascular tissue construct into the nanosilicatemodified GelMA hydrogel, the osteogenic potential of mesenchymal stem cells and bone mineralization were further enhanced in the presence of osteoinducting factors and VEGF, suggesting that this vascularized $3 \mathrm{D}$ bone tissue construct could be potentially used as a bone transplant for bone regeneration [68]. A more stable and large-scale $3 \mathrm{D}$ vascularized tissue construct can be bioprinted by using a blended bioink consisting of GelMA hydrogel, sodium alginate and PEGTA, offering great promise for tissue repair and regeneration [76]. For cardiac tissue engineering, other materials such as reduced graphene oxide or carbon nanotubes were incorporated into GelMA hydrogel to increase its electrical conductivity for maturation of cardiomyocytes. Upon electrical stimulation, cardiomyocytes encapsulated in such hybrid hydrogels demonstrate higher expression of cardiac markers and faster spontaneous beating than those encapsulated in the pristine GelMA hydrogels $[87,88]$. GelMA/ black phosphorus hybrid hydrogel could also be a promising scaffold for such application [89]. For skin regeneration, it was shown that GelMA/MeHA hybrid hydrogels loaded with adipose-derived stem cells exhibit an excellent angiogenic potential for wound healing [90]. Additionally, dopamine (a neurotransmitter that regulates neuronal development) can be added into GelMA hydrogel to improve neuronal differentiation of neural stem cells [91]. To promote sutureless peripheral nerve repair, GelMA hydrogel can be functionalized with methacryloyl-substituted tropoelastin to enhance its adhesive strength to nerve tissue, which is approximately 15 -fold higher than that of conventional fibrinbased adhesives [72]. With the incorporation of methacryloyl-substituted recombinant human tropoelastin into a visible light-crosslinked GelMA hydrogel, it exhibits excellent adhesive properties that are capable of promoting sutureless wound healing [92].

\section{Drug delivery}

GelMA hydrogels have also been used in controlling the delivery of drugs and growth factors for therapeutic applications. For instance, GelMA/polyacrylamide hybrid hydrogel has been used to control the release of sodium nafcilin monohydrate, an antibiotic against Staphylococcus sp. bacteria [93]. Moreover, by integrating a dielectrophoresis system with gold microparticleladen GelMA hydrogels, the controlled release of drugs was achieved with a concentration gradient for highthroughput drug screening applications [34]. On the other hand, by incorporating chitosan nanoparticles into GelMA hydrogel, which provides a sustained release of basic fibroblast growth factor, this hybrid hydrogel can be used for angiogenesis in various diseases, particularly ischemic diseases [75]. Furthermore, a coacervate microdroplet-laden hydrogel consisting of GelMA and oxidized, methacrylated alginate was developed for the controlled release of growth factors to the encapsulated stem cells for stem-cell-based therapies [63] (Figure 3C). Last but not least, GelMA hydrogel functionalized with polyethylenimine and graphene oxide nanosheets has been used as a nonviral gene delivery system to exert gene therapy for the treatment of acute myocardial infarction [94].

\section{Biosensing}

For biosensing applications, GelMA hydrogel was functionalized with nickel nanoparticles and reduced graphene oxide to create a glucose sensor [95]. These nanomaterials enhance electron transfer capacity and glucose catalytic oxidation activity for electrochemical sensing of glucose. This sensor was successfully used to determine the concentration of glucose in human blood serum [95]. On the other hand, an intelligent wound dressing consisting of a GelMA hydrogel encapsulating a bacterial infection-responsive lipid vesicle and antimicrobials was developed for fluorescent detection and inhibition of pathogenic bacteria (e.g., S. aureus and Pseudomonos aeruginosa) [64] (Figure $3 D$ ). In the presence of pathogenic bacteria, the lipid vesicles were hydrolyzed by the enzymes or toxins secreted by the bacteria, thereby releasing detectable fluorescence signal (an indicator for infection) and antimicrobials. This sensor was used to detect and treat drug-resistant bacterial infections, which will potentially be useful for future clinical therapies.

\section{MeHA hydrogel}

$\mathrm{HA}$ is a biocompatible nonsulfated glycosaminoglycan composed of repeating disaccharide units of $\mathrm{N}$-acetylglucosamine and D-glucuronic acid that is abundantly present in large amounts in tissues, including cartilage, neuron and skin [96]. HA contains target sequences of hyaluronidases that can promote remodeling of the cellular microenvironment [97], and can specifically bind to hyaluronan receptors (CD44 and CD168), which are highly expressed by 
most tumor cells [98]. However, HA hydrogel exhibits poor mechanical properties and rapid degradation [99], limiting its use in biomedical applications. To this end, manipulating methacrylation of $\mathrm{HA}$ is essential to achieve its optimum mechanical properties and control its degradation rate $[99,100]$, making MeHA hydrogel suitable for various biomedical applications, including tissue engineering and regenerative medicine, drug delivery and biosensing $[98,101,102]$.

\section{Synthesis of photo-crosslinkable MeHA hydrogel}

To synthesize MeHA, the first step is to methacrylate HA (substitution of methacryloyl groups on the hydroxyl and reactive amine groups of $\mathrm{HA}$ ) in deionized water $(\mathrm{pH}=8.0-8.5)$ at $4^{\circ} \mathrm{C}$ for producing the MeHA pre-polymer solution $[98,103]$. Subsequently, the pre-polymer solution is dialyzed using a dialysis tubing with MW cut-offs that range from 6 to $8 \mathrm{kDa}$ for 2-3 days to remove all the MA byproducts and unreacted MA $[98,104]$. Finally, the MeHA pre-polymers are produced by freeze-drying the dialyzed solution. These pre-polymers $(0.25$ to $4.0 \%)(w / v)$ can be used to fabricate MeHA hydrogel constructs in the presence of UV light and Irgacure 2959 through various microfabrication strategies (e.g., electrospinning, micromolding and bioprinting) $[105,106]$. Similar to GelMA hydrogel, the mechanical properties of MeHA hydrogel can be tuned by varying the pre-polymer concentration or light exposure duration [106,107]. For instance, elastic modulus of MeHA hydrogel was increased from 1.3 to $10.6 \mathrm{kPa}$ when the pre-polymer concentration was increased from 1 to $3 \%$ (w/v) [106] (Table 3). Besides mechanical properties, the degradation rate of MeHA hydrogel can be adjusted by varying its pre-polymer concentration. When the pre-polymer concentration was increased from 1 to $3 \%$, enzymatic degradation of MeHA hydrogel was decreased from 100 to $50 \%$ after 1 day of incubation in hyaluronidase solution [106]. High degradability of MeHA hydrogel is imperative for cell remodeling in 3D tissueengineered constructs [103].

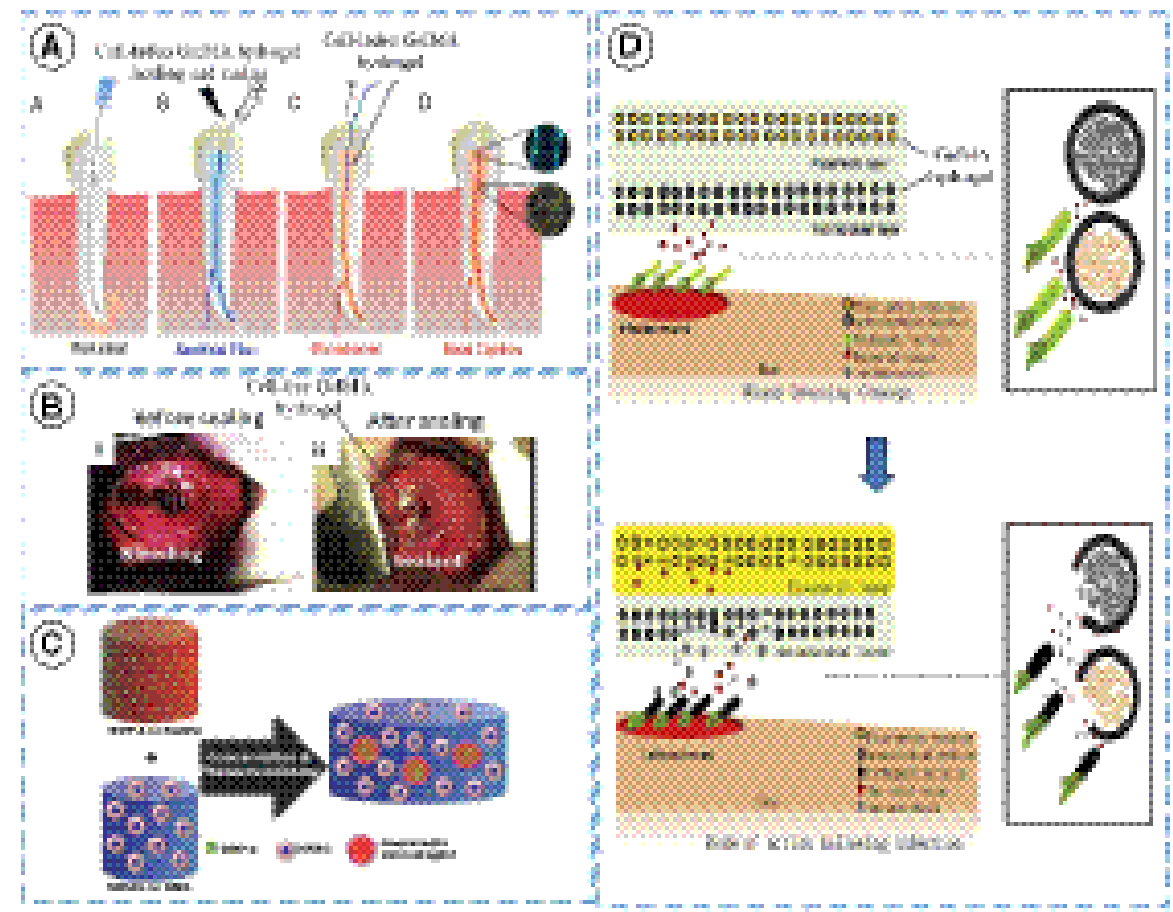

Figure 3. Biomedical applications of GelMA hydrogel. (A) Tooth regeneration; adapted with permission from [62]. (B) Air leak sealant; adapted with permission from [67] ๔ Elsevier (2017). (C) Growth factor delivery for stem-cell based therapy; reproduced with permission from [63] @ John Wiley \& Sons (2015). (D) Sensor in wound dressing for the detection and inhibition of pathogenic bacteria; adapted with permission from [64] ๔ Elsevier (2018).

GeIMA: Gelatin methacryloyl; hMSCs: Human mesenchymal stem cells; OMA: Oxidized, methacrylated alginate.

\section{RECENT BIOMEDICAL APPLICATIONS OF PHOTO- CROSSLINKABLE MEHA HYDROGEL}

Drug delivery for cancer therapy

MeHA hydrogel has a high tumor targeting capability. For example, HA is a natural ligand to hyaluronan receptor (e.g., CD44), which is overexpressed in various types of tumors, suggesting its great potential in drug delivery and cancer therapy [108]. UV light-crosslinked MeHA hydrogel was incorporated with upconversion nanoparticles to deliver carboxyl group-modified zinc phthalocyanines (a photosensitizer), which can generate an abundance of singlet oxygen upon activation using near-infrared light to kill gastric cancer cells [109]. MeHA hydrogel was also incorporated with drug-loaded mesoporous silica nanoparticles (a sonosensitizer) to achieve a chemotherapy-sonodynamic therapy for the treatment of breast cancer [101] (Figure 4A). To further increase tumor target and drug delivery efficiency, endosome membrane components from cancer cells have been used to encapsulate
MeHA hydrogel loaded with doxorubicin. This enables the drug-loaded hydrogel to be uptaken easily and specifically by cancer cells for effective cancer chemotherapy [108] (Figure 4B).

\section{Tissue engineering}

As HA is a major ECM component for CNS development, UV light-crosslinked MeHA hydrogels were used to engineer $3 \mathrm{D}$ in vitro CNS normal and disease tissue models for the study of CNS diseases and regeneration [110]. MeHA hydrogels have also been used to generate tissue engineered cartilageand bone-like tissue constructs for cartilage and bone regeneration, respectively $[104,106]$. To further improve biofunctionality of MeHA hydrogel, incorporating other organic or inorganic components into it can enhance its mechanical and chemical properties. For nerve repair, combination of MeHA hydrogel with puramatrix peptide hydrogel could improve neurite extension or outgrowth [111]. Moreover, MeHA hydrogels have been electrospun into aligned nanofibers and incorporated with poly-lactic-co-glycolic 

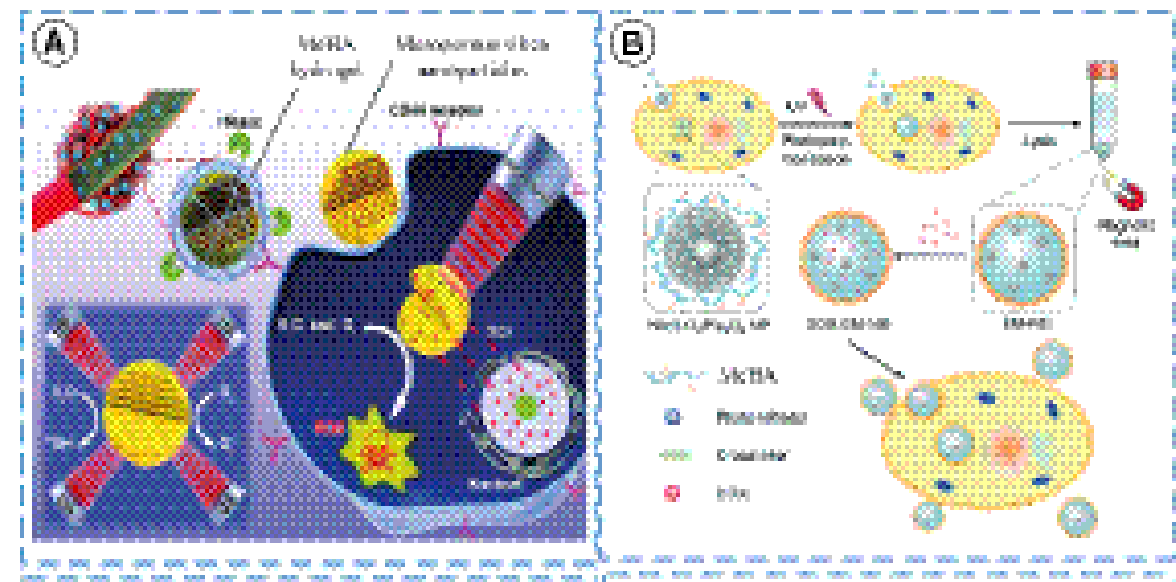

(C)

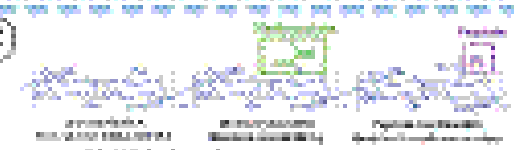

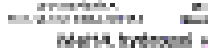
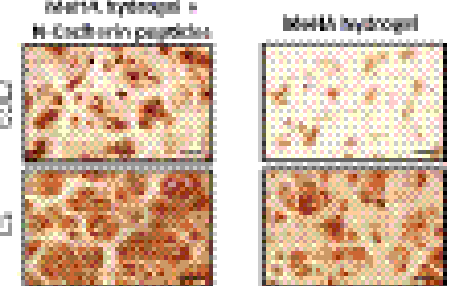

11
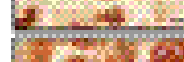

3

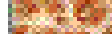

Pathos

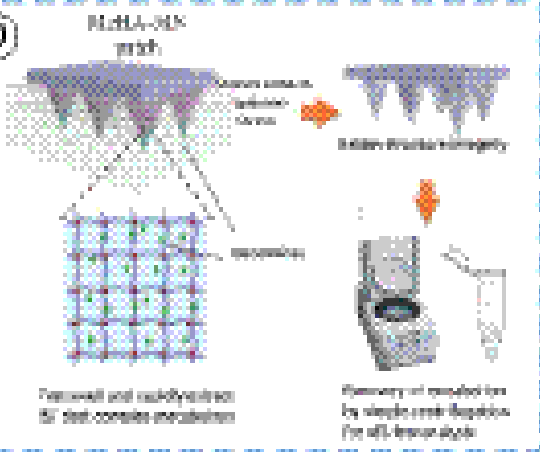

Figure 4. Biomedical applications of MeHA hydrogel. (A) Synergistic cancer chemotherapy and sonodynamic therapy; adapted with permission from [101] @ Royal Society of Chemistry (2017). (B) Targeted cancer chemotherapy; adapted with permission from [108]. (C) Cartilage tissue engineering; adapted with permission from [98]. (D) Microneedle-based extraction of skin ISF for metabolite sensing; adapted with permission from [102] @ John Wiley \& Sons (2017). COL2: Collagen type 2; CS: Chondroitin sulphate; Dox: Doxorubicin; EM-NG: Endosome membranenanogel; HAase: Hyaluronidase; ISF: Interstitial fluid; MeHA: Methacrylated hyaluronic acid; MN: Microneedle; ROS: Reactive oxygen species; UV: Ultraviolet.

$>$ microspheres to control the release of nervous growth factor for peripheral nerve repair and regeneration [105]. Cells (chondrocytes or mesenchymal stem cells) are encapsulated in MeHA hydrogels functionalized with methacrylated chondroitin sulfate, devitalized cartilage microparticles or $\mathrm{N}$-cadherin mimetic peptides (Figure 4C). These encapsulated cells demonstrate increased expression of cartilage markers and deposition of collagen type II compared with those encapsulated in pristine MeHA hydrogels $[98,112,113]$. In addition, MeHA/GelMA hybrid hydrogel has been used to engineer $3 \mathrm{D}$ in vitro fibrotic heart valve tissue models for understanding the process of fibrotic remodeling and calcification in heart valve diseases $[107,114,115]$.

\section{Biosensing}

Due to the excellent hydrating functions of HA, MeHA hydrogel has been used to make a microneedle patch for extraction of skin inter- stitial fluid (ISF) for biosensing applications. This microdevice is capable of detecting and analyzing the metabolites in ISF, such as cholesterol and glucose, in a rapid and minimally invasive manner [102] (Figure 4D).

\section{FUTURE PERSPECTIVE}

$3 \mathrm{D}$ bioprinting techniques (e.g., inkjet printing, extrusion printing, laser-assisted printing and stereolithography) are valuable tools for the fabrication of 3D tissue-engineered constructs and hydrogel-based biomedical devices due to their ability to precisely fabricate external geometries and complex internal architectures [116]. Specifically, stereolithography is the most advanced technique for printing photo-crosslinkable hydrogels. In fact, stereolithography produces complex 3D structures using a layer-by-layer approach by spatially controlling photopolymerization of photo-crosslinkable hydrogel solutions under UV or visible light based on images generated from computer-aided design software. Stereolithography has many advantages over other bioprinting techniques. First, it is nozzle-free, indicating that cells are not subjected to external force during printing of cell-laden hydrogel constructs, which ensures high cell viability. Moreover, compared with other bioprinting techniques, it has a relatively faster printing speed, greater vertical printing ability and higher resolution at minimal cost. Stereolithography has been used to fabricate a 3D detoxifier with four layers of liver-mimetic structure made up of PEGDA-based hydrogel [45]. Additionally, stereolithography has demonstrated its capability to print 3D in vitro cell-laden tissue models made up of GelMA-based hydrogel, such as a tumor angiogenesis model, skeletal muscle model and tendon-to-bone insertion model [117]. However, most of the stereolithographybased bioprinting systems still rely on harmful UV light. Therefore, many efforts have been devoted to printing 3D photocrosslinkable hydrogel structures for biomedical applications using stereolithography under visible light.

There are many challenges associated with the clinical translation of photocrosslinkable hydrogels that need to be addressed. As aforementioned, the sole PEGDA hydrogel is lacking strong cell adhesion peptides, making it less effective for $3 \mathrm{D}$ cell culturing. Therefore, more studies should focus on combining PEGDA with other materials with strong cell adhesive properties (e.g., GelMA, HA, etc) to produce a hybrid ECM-mimicking hydrogel. This can effectively help to rebuild new ECM for tissue engineering and drug delivery applications. Recently, it was reported that type B GelMA hydrogel that contains bovine gelatin exhibits good biocompatibility. However, type A GelMA that contains porcine gelatin may induce inflammatory reactions [118]. Therefore, further biosafety assessments on GelMA-based tissue-engineered constructs are required prior to clinical applications to minimize biosafety concerns. Other challenges in clinical translation of GelMAbased tissue engineered constructs are potential disease transmission associated with the use of animal-based materials and batch-to-batch variations. Hence, it is imperative to develop a human-derived gelatin as an alternative to animal-based gelatin. 
In addition, a standardized protocol for synthesis of GeIMA should also be established and implemented across all laboratories. MeHA hydrogel can only be used to culture limited types of cells (e.g., chondrocytes, neurons), compared with GelMA hydrogel, due to the inherent properties of HA. Therefore, incorporating functional peptide (e.g., RGD peptides) into MeHA is important, in particular to achieve a broader range of tissue engineering applications.

Photo-crosslinkable tissue-engineered hydrogel constructs have not been fully applied in clinical applications due to restrictions with regard to their biosafety concerns associated with exposure to UV light, for example oxidative damage to cellular DNA, cancer formation and accelerated tissue aging, limiting their use as implants for tissue repair and regeneration [119]. To address these issues, visible light with wavelengths of 450-550 nm could be an alternative light source for crosslinking of photo-crosslinkable hydrogels. Visible light can deeply penetrate tissues with relatively low energy, making it suitable for the development of in situ injectable hydrogels for in vivo applications in a minimally invasive manner. Additionally, near-infrared irradiation, which is harmless and able to penetrate tissues, could also be used to induce the in situ formation of hydrogel for various biomedical applications (e.g., cancer therapy). Biosafety assessments of both cell-free and cell-laden photo-crosslinkable hydrogels are important to ensure the safe and effective use of hydrogels.

\section{CONCLUSION}

In conclusion, photo-crosslinkable hydrogels such as PEGDA, GelMA and MeHA can be readily photo-crosslinked to achieve desirable mechanical properties and biological responses in a spatiotemporal manner, making them a versatile platform for various biomedical applications (e.g., tissue engineering, drug delivery and cancer therapy). In particular, PEGDA hydrogel is highly tunable and is more easily synthesized than GeIMA and MeHA, and its mechanical and physical properties can be readily tuned by varying the concentration and molecular weight of the polymers. These properties make it highly suitable for a wide range of biomedical applications. As for the GelMA hydrogel, its tunable mechanical properties and the presence of functional RGD peptides make it an essential material for the development of various types of 3D tissueengineered implants and in vitro tissue models for regenerative medicine. MeHA hydrogel has high tumor target efficiency, and hence it is widely used for cancer therapy. With many opportunities and remaining challenges, we envision that there will be more studies focusing on photo-crosslinkable hydrogels dedicated to biomedical applications in the future.

\section{AUTHOR CONTRIBUTIONS}

JRC and KWY contributed to the conception and design of the work. All authors participated in the discussions and writing of the manuscript. All authors reviewed and approved the final version of the manuscript for submission.

\section{FINANCIAL \& COMPETING INTERESTS DISCLOSURE}

This work was supported by Michael Smith Foundation for Health Research; and Office of Vice President Research, University of Calgary. The authors have no other relevant affiliations or financial involvement with any organization or entity with a financial interest in or financial conflict with the subject matter or materials discussed in the manuscript apart from those disclosed.

No writing assistance was utilized in the production of this manuscript.

\section{OPEN ACCESS}

This work is licensed under the AttributionNonCommercial-NoDerivatives 4.0 Unported License. To view a copy of this license, visit http://creativecommons.org/licenses/by-nc$\mathrm{nd} / 4.0 /$

\section{REFERENCES}

\section{Papers of special note have been highlighted as:} - of interest; • of considerable interest

1. Choi JR, Yong KW, Tang R et al. Lateral flow assay based on paper-hydrogel hybrid material for sensitive pointof-care detection of dengue virus. Adv. Healthcare Mater. 6(1), (2017).

2. Li J, Mo L, Lu C-H, Fu T, Yang H-H, Tan W. Functional nucleic acid-based hydrogels for bioanalytical and biomedical applications. Chem. Soc. Rev. 45(5), 1410-1431 (2016).

\section{EXECUTIVE SUMMARY}

\section{PHOTOPOLYMERIZATION REACTION}

$\otimes$ There are two photopolymerization reactions: free radical initiated chaingrowth polymerization and bio-orthogonal click reaction.

\ Photo-crosslinkable hydrogels such as poly(ethylene glycol) diacrylate (PEGDA), gelatin methacryloyl (GelMA) and methacrylated hyaluronic acid (MeHA), are generally synthesized through free radical initiated chain-growth polymerization.

\section{PHOTOINITIATORS FOR}

\section{PHOTOPOLYMERIZATION}

\Commonly used radical photoinitiators for photopolymerization of PEGDA, GeIMA and
MeHA are Irgacure 2959, lithium phenyl2,4,6-hydroxyethyl)propionamide and Eosin-Y.

\ Both UV light-sensitive photoinitiators (e.g., Irgacure 2959) and visible lightsensitive photoinitiators (e.g., Eosin-Y) have some advantages and disadvantages.

\section{PEGDA HYDROGEL}

$\triangle$ PEGDA hydrogel is mainly used in drug delivery, as it acts as a stable medium to protect encapsulated drugs for long-term controlled release of drugs, due to its low degradation rate.

\section{GELMA HYDROGEL}

\ GelMA hydrogel contains functional arginine, glycine, and aspartat peptides, making it an essential material in tissue engineering especially for the development of various types of biomimetic 3D tissueengineered implants and in vitro tissue models.

\section{MEHA HYDROGEL}

$\otimes$ MeHA hydrogel has a high tumor target efficiency due to its ability to bind hyaluronan receptors that are highly expressed by tumor cells, and hence it is broadly used for cancer therapy. 
> 3. Lee TT, García JR, Paez Jl et al. Light-triggered in vivo activation of adhesive peptides regulates cell adhesion, inflammation and vascularization of biomaterials. Nat. Mater. 14(3), 352 (2015).

4. Vashist A, Vashist A, Gupta Y, Ahmad $S$. Recent advances in hydrogel base drug delivery systems for the human body. J. Mater. Chem. B 2(2), 147-166 (2014).

5. Vashist A, Kaushik A, Alexis K et al. Bioresponsive injectable hydrogels for on-demand drug release and tissue engineering. Curr. Pharm. Des. 23(24), 3595-3602 (2017)

6. Highley CB, Prestwich GD, Burdick JA Recent advances in hyaluronic acid hydrogels for biomedical applications. Curr. Opin. Biotechnol. 40, 35-40 (2016).

7. Caló E, Khutoryanskiy VV. Biomedical applications of hydrogels: a review of Polym. J. 65, 252-267 (2015).

8. Yue $K$, Trujillo-De Santiago $G$, Alvarez MM, Tamayol A, Annabi N, Khademhosseini A. Synthesis, properties, and biomedical applications of gelatin methacryloyl (GeIMA) hydrogels. Biomaterials 73, 254-271 (2015).

9. Yuan M, Bi B, Huang J, Zhuo R, Jiang X. Thermosensitive and photocrosslinkable hydroxypropyl chitin-based hydrogels for biomedical applications. Carbohydr. Polym. 192, 10-18 (2018).

10. Wang R, Yang Z, Luo J, Hsing I-M, Sun F. B12-dependent photoresponsive protein hydrogels for controlled stem
cell/protein release. Proc. Natl Acad. Sci. USA 114(23), 5912-5917 (2017).

11. Zhao $X$, Lang $Q$, Yildirimer $L$ et al. Photocrosslinkable gelatin hydrogel for epidermal tissue engineering. Adv.
Healthcare Mater. 5(1), 108-118 (2016).

12. Nguyen $Q T$, , Hwang $Y$, Chen AC, Varghese S, Sah RL. Cartilage-like mechanical crylate hydrogels. Biomaterials 33(28), 6682-6690 (2012).

13. Yang $C$, Wang $X$, Yao $X$, Zhang $Y$, Wu $W$ Jiang $X$. Hyaluronic acid nanogels with enzyme-sensitive cross-linking group for drug delivery. J. Control. Rel. 205, 206-217 (2015).

14. Ouyang L, Highley CB, Sun W, Burdick JA. A generalizable strategy for the $3 \mathrm{D}$ bioprinting of hydrogels from nonviscous photo-crosslinkable inks. $A d v$. Mater. 29(8), (2017)

15. Truong VX, Li F, Forsythe JS. Photolabile hydrogels responsive to broad spectrum visible light for selective ce release. ACS Applied Mat. Interfaces 9(38), 32441-32445 (2017).

16. Pereira RF, Bártolo PJ. 3D photo-fabrication for tissue engineering and drug delivery. Engineering 1(1), 090-112 (2015).

17. Lin CC, Ki CS, Shih H. Thiol-norbornene photoclick hydrogels for tissue engineering applications. J. Appl. Polym. Sci. 132(8), (2015).

18. Annabi N, Tamayol A, Uquillas JA et al. 25th anniversary article: rational design and applications of hydrogels in regenerative medicine. Adv. Mater. 26(1), 85-124 (2014)

19. Azagarsamy MA, Anseth KS. Bioorthogonal click chemistry: an indispensable tool to create multifaceted cell cultur scaffolds. ACS Macro. Lett. 2(1), 5-9 (2012).

20. Tibbitt MW, Kloxin AM, Sawicki LA Anseth KS. Mechanical properties and degradation of chain and step-polymerzed photodegradable hydrogels. Macromolecules 46(7), 2785-2792 (2013).

21. Jiang $Y$, Chen J, Deng C, Suuronen EJ, Zhong Z. Click hydrogels, microgels and nanogels: emerging platforms for drug delivery and tissue engineering. Biom
terials 35(18), 4969-4985 (2014).

22. Kyburz KA, Anseth KS. Three-di- mensional hMSC motility within peptide-functionalized PEG-based hydrogels of varying adhesivity and crosslinking density. Acta Biomater 9(5), 6381-6392 (2013)

23. Shih $\mathrm{H}$, Lin CC. Visible-light-mediated thiol-Ene hydrogelation using eosin- $Y$ as the only photoinitiator. Macromo Rapid Commun. 34(3), 269-273 (2013).

24. Fairbanks $B D$, Schwartz MP, Halevi $A E$, Nuttelman CR, Bowman CN, Anseth KS. A versatile synthetic extracellular matrix mimic via thiol-norbornene photopolymerization. Adv. Mater. 21(48), 5005-5010 (2009)

25. Espeel P, Goethals F, Du Prez FE. One-pot multistep reactions based on thiolactones: extending the realm of thiol-ene chemistry in polymer synthesis. Am. Chem. Soc. 133(6), 1678-1681 (2011).

26. Nguyen KT, West JL. Photopolymerizable hydrogels for tissue engineering applications. Biomaterials 23(22), 4307-4314 (2002)

27. Rouillard AD, Berglund CM, Lee JY et al. Methods for photocrosslinking alginate hydrogel scaffolds with high cell viability. Tissue Eng., Part C 17(2), 173-179 (2010)

28. Billiet T, Gevaert E, De Schryver T, Cornelissen M, Dubruel P. The 3D printing of gelatin methacrylamide cell-laden tissue-engineered constructs with high cell viability. Biomaterials 35(1), 49-62 (2014).

29. Hu J, Hou Y, Park H et al. Visible light crosslinkable chitosan hydrogels for tissue engineering. Acta Biomater. 8(5), 1730-1738 (2012)

30. Ruskowitz ER, Deforest CA. Photoresponsive biomaterials for targeted drug delivery and 4D cell culture. Nat. Rev. Mater. 3, 17087 (2018).

31. Ma Y, Ji Y, Zhong T et al. Bioprinting-based PDLSC-ECM screening for in vivo repair of alveolar bone defect using cell-laden, injectable and photoSci. Eng. 3(12), 3534-3545 (2017).

32. Munoz-Pinto DJ, Jimenez-Vergara AC, Gharat TP, Hahn MS. Characterization of sequential collagen-poly (ethylene glycol) diacrylate interpenetrating networks and initial assessment of thei potential for vascular tissue engineering. Biomaterials 40, 32-42 (2015).

33. Chen G, Kawazoe N, Ito Y. Photo-crosslinkable hydrogels for tissue engineering applications. In: Photochemistry for Biomedical Applications. Springer, 277-300 (2018).

34. Ahadian S, Ramón-Azcón J, Estil M, Obregón R, Shiku H, Matsue T. Facile and rapid generation of $3 \mathrm{D}$ chemical gradients within hydrogels for high-throughput drug screening applications. Biosens. Bioelectron. 59, 166-173 (2014).

35. Schesny MK, Monaghan M, Bindermann $\mathrm{AH}$ et al. Preserved bioactivity and tunable release of a SDF1-GPVI bi-specific protein using photo-crosslinked PEGda hydrogels. Biomaterials 35(25) 7180-7187 (2014).

36. Tian F, Lyu J, Shi J, Tan F, Yang M. A polymeric microfluidic device integrated with nanoporous alumina membranes for simultaneous detection of multiple foodborne pathogens. Sens. Actuators $B$ 225, 312-318 (2016)

37. Marchioli G, Zellner L, Oliveira C et al. Layered PEGDA hydrogel for islet of Langerhans encapsulation and improvement of vascularization. J. Mater. Sci. 28(12), 195 (2017).

38. Clark EA, Alexander MR, Irvine DJ et al. 3D printing of tablets using inkjet with UV photoinitiation. Int. J. Pharm. 529(1-2), 523-530 (2017).

39. Lim WS, Chen K, Chong TW et al. A bilayer swellable drug-eluting ureteric stent: Localized drug delivery to treat
urothelial diseases. Biomaterials 165 ,
25-38 (2018)

40. Liu S, Yeo DC, Wiraja C, Tey HL, Mrksich $M$, Xu C. Peptide delivery with poly (ethylene glycol) diacrylate microneedles through swelling effect. Bioeng. Trans Med. 2(3), 258-267 (2017)

PEGDA hydrogel was made into a microneedle patch to deliver drug for the treatment of skin diseases.

41. Zhai X, Ruan C, Ma Y et al. 3D-bioprinted osteoblast-laden nanocomposit hydrogel constructs with induced microenvironments promote cell viaboth in vitro and in vivo. Adv. Sci. 5(3), 1700550 (2017).

42. Son KJ, Gheibi P, Stybayeva G, Rahimian A, Revzin A. Detecting cell-secreted growth factors in microfluidic devices using bead-based biosensors. Mi-
crosyst. Nanoeng. 3, 17025 (2017)

43. Zeng Z, Mo X-M, He C, Morsi $Y$, El-Hamshary H, El-Newehy M. An in situ forming tissue adhesive based on poly (ethylene glycol)-dimethacrylate and thiolated chitosan through the Michae reaction. J. Mater. Chem. B 4(33), 5585-5592 (2016).

44. Jabbari E, Sarvestani SK, Daneshian L, Moeinzadeh S. Optimum 3D matrix stiffness for maintenance of cancer stem cells is dependent on tissue origin of cancer cells. PloS One 10(7), e0132377 (2015).

45. Gou M, Qu X, Zhu W et al. Bio-inspired detoxification using 3D-printed hydrogel nanocomposites. Nat. Commun. 5, 3774 (2014).

\section{PEGDA hydrogel was made into a 3D} structure mimicking liver lobules and loaded with polydiacetylene nanoparticles to effectively detect, capture and remove toxins from the human body.

46. Hutson CB, Nichol JW, Aubin $\mathrm{H}$ et al. Synthesis and characterization of tunable poly (ethylene glycol): gelatin methacrylate composite hydrogels. Tissue Eng., Part A 17(13-14), 1713-1723 (2011).

47. Aycan D, Alemdar N. Development of $\mathrm{pH}$-responsive chitosan-based hydroge modified with bone ash for controlled release of amoxicillin. Carbohydr. Polym. 184, 401-407 (2017)

48. Guarino V, Alvarez-Perez MA, Borriello A, Napolitano T, Ambrosio L. Conductive PANi/PEGDA macroporous hydrogels for nerve regeneration. Adv. Healthcare Mater. 2(1), 218-227 (2013).

49. Zhu W, George JK, Sorger VJ, Zhang LG. 3D printing scaffold coupled with low level light therapy for neural tissue regeneration. Biofabrication 9(2), 025002 (2017)

50. Bae JH, Lee JM, Chung BG. Hydrogel-encapsulated 3D microwell array for 11(1), 015019 (2016).

51. Hao Y, Lin CC. Degradable thiol-acrylate hydrogels as tunable matrices for three-dimensional hepatic culture. $J$. Biomed. Mater. Res., Part A 102(11), 3813-3827 (2014)

52. Nachlas $A L$, Li S, Jha $R$, Singh $M, X$ C. Davis ME. Human iPSC-derived mesenchymal stem cells matured into valve interstitial-like cells using PEGDA hydrogels. Acta Biomater. 11(1), 015019 (2018).

53. Shohatee D, Keifer J, Schimmel N, Mohanty S, Ghosh G. Hydrogel-based suspension array for biomarker detection using horseradish peroxidase-mediated silver precipitation. Anal. Chim. Acta 999, 132-138 (2018).

54. Kadilak AL, Rehaag JC, Harrington CA Shor LM. A 3D-printed microbial cell culture platform with in situ PEGDA hydrogel barriers for differential substrate (2017).

55. Klotz BJ, Gawlitta D, Rosenberg AJ, Malda J, Melchels FP. Gelatin-methacryloyl hydrogels: towards biofab-

rication-based tissue repair. Trends Biotechnol. 34(5), 394-407 (2016).

56. Lai J-Y, Li Y-T. Functional assessment of cross-linked porous gelatin hydrogels for bioengineered cell sheet carriers. Biomacromolecules 11(5), 1387-1397 (2010)

57. Liu Y, Chan-Park MB. A biomimetic hydrogel based on methacrylated dextran-graft-lysine and gelatin for 3D smooth muscle cell culture. Biomaterials 31(6), 1158-1170 (2010).

58. Xing Q, Yates K, Vogt C, Qian Z, Frost MC Zhao $F$. Increasing mechanical strength of gelatin hydrogels by divalent metal ion removal. Sci. Rep. 4, 4706 (2014).

59. Van Den Bulcke Al, Bogdanov B, De Rooze N, Schacht EH, Cornelissen M, 
et al. Photocrosslinkable gelatin/tropoe lastin hydrogel adhesives for peripheral nerve repair. Tissue Eng., Part A (2018). doi:10.1089/ten.TEA.2017.0502

73. Fan L, Liu C, Chen X et al. Directing nduced pluripotent stem cell derived neural stem cell fate with a three-dimensional biomimetic hydrogel for spinal cord injury repair. ACS Appl. Mater. Interfaces 10(21), 17742-17755 (2018).

74. Zhao $X$, Sun $X$, Yildirimer $L$ et al. Cell infiltrative hydrogel fibrous scaffolds for accelerated wound healing. Acta Biomater. 49, 66-77 (2017).

75. Modaresifar K, Hadjizadeh A, Niknejad H. Design and fabrication of GelMA chitosan nanoparticles composite hydrogel for angiogenic growth factor delivery Artif Cells Nanomed Bictor 1-10 (2017)

76. Jia W, Gungor-Ozkerim PS, Zhang YS et al. Direct 3D bioprinting of perfusable vascular constructs using a blend bioin Biomaterials 106, 58-68 (2016).

77. Paul A, Manoharan V, Krafft D et al. Nanoengineered biomimetic hydrogels for guiding human stem cell osteogenesis in three dimensional microenvironments. $J$. Mater. Chem. B 4(20), 3544-3554 (2016)

78. Levato R, Webb WR, Otto IA et al. The bio in the ink: cartilage regeneration with bioprintable hydrogels and articula cartilage-derived progenitor cells. Acta Biomater. 61, 41-53 (2017)

79. Zhang YS, Davoudi F, Walch P et al. Bioprinted thrombosis-on-a-chip. Lab Chip 16(21), 4097-4105 (2016)

80. Bhise NS, Manoharan V, Massa S et al. A liver-on-a-chip platform with bioprinted hepatic spheroids. Biofabrication 8(1) 014101 (2016)

81. Erkoc $P$, Seker F, Bagci-Onder T, Kizilel S. Gelatin methacryloyl hydrogels in the absence of a crosslinker as 3D glioblastoma multiforme (GBM)-mimetic microenvironment. Macromol. Biosci. 18(3) (2018).

82. Levett PA, Melchels FP, Schrobback K, Hutmacher DW, Malda J, Klein TJ. A biomimetic extracellular matrix for cartilage tissue engineering centered on photocurable gelatin, hyaluronic acid and chondroitin sulfate. Acta Biomater. 10(1), 214-223 (2014).

83. Meinert C, Schrobback K, Hutmacher DW, Klein TJ. A novel bioreactor system for biaxial mechanical loading enhances the properties of tissue-engineered human cartilage. Sci. Rep. 7(1), 16997 (2017)

84. Brown GC, Lim KS, Farrugia BL, Hooper GJ, Woodfield TB. Covalent incorporation of heparin improves chondrogenesis in photocurable gelatin-methacryloyl hydrogels. Macromol. Biosci. 17(12), (2017).

85. Mouser VH, Melchels FP, Visser J, Dher WJ, Gawlitta D, Malda J. Yield stress determines bioprintability of hydrogels based on gelatin-methacryloyl and gellan gum for cartilage bioprinting. Biofabrication 8(3), 035003 (2016).

86. Wenz A, Borchers K, Tovar GE, Kluger PJ. Bone matrix production in hydroxyapatite-modified hydrogels suitable for bon bioprinting. Biofabrication 9(4), 044103 (2017).

87. Shin SR, Zihlmann C, Akbari $M$ et al Reduced graphene oxide-gelMA hybrid hydrogels as scaffolds for cardiac tissu engineering. Small 12(27), 3677-3689 (2016).

88. Ahadian S, Yamada S, Ramón-Azcón $\mathrm{J}$ et al. Hybrid hydrogel-aligned carbon nanferube scaffolds to enhance cardiac Biomater. 31, 134-143 (2016)

89. Choi JR, Yong KW, Choi JY et al. Black phosphorus and its biomedical applications. Theranostics 8(4), 1005-1026 (2018).

90. Eke G, Mangir N, Hasirci N, Macneil $S$, Hasirci V. Development of a UV crosslinked biodegradable hydroge containing adipose derived stem cells to promote vascularization for skin wounds and tissue engineering. Biomaterials 129,
188-198 (2017).

91. Zhou X, Cui H, Nowicki M et al. Three-dimensional-bioprinted dopamine-based matrix for promoting neural regeneration. ACS Appl. Mater. Interfaces 10(10) 8993-9001 (2018).

92. Annabi N, Rana D, Sani ES et al. Engineering a sprayable and elastic hydroge adhesive with antimicrobial properties for wound healing. Biomaterials 139 , 229-243 (2017).

93. Serafim A, Tucureanu C, Petre D-G et al. One-pot synthesis of superabsorbent hybrid hydrogels based on methacrylamide gelatin and polyacrylamide. Effortless control of hydrogel properties throug composition design. New J. Chem. 38(7) 3112-3126 (2014).

94. Paul A, Hasan A, Kindi HA et al. Injectable graphene oxide/hydrogel-based angiogenic gene delivery system for vasculogenesis and cardiac repair. ACS Nano 8(8), 8050-8062 (2014).

95. Darvishi S, Souissi M, Kharaziha M Karimzadeh F, Sahara R, Ahadian S. Gelatin methacryloyl hydrogel for glucose biosensing using Ni nanoparticles-reduced graphene oxide: an experimenta and modeling study. Electrochim. Acta 261, 275-283 (2018)

96. Burdick JA, Prestwich GD. Hyaluronic acid hydrogels for biomedical applications. Adv. Mater. 23(12), (2011).

97. Lei Y, Gojgini S, Lam J, Segura T. The spreading, migration and proliferation of mouse mesenchymal stem cells cultured inside hyaluronic acid hydrogels. Biomate rials 32(1), 39-47 (2011).

98. Bian L, Guvendiren M, Mauck RL, Burdick JA. Hydrogels that mimic developmentally relevant matrix and $\mathrm{N}$-cadherin interactions enhance MSC chondrogenesis. Proc. Natl Acad. Sci. USA 110(25), 10117-10122 (2013).

99. Tavsanli B, Okay 0 . Mechanically strong hyaluronic acid hydrogels with an interpenetrating network structure. Eur. Polym J. 94, 185-195 (2017)

100.Sahoo S, Chung C, Khetan S, Burdick JA. Hydrolytically degradable hyaluronic acid hydrogels with controlled tempora structures. Biomacromolecules 9(4), 1088-1092 (2008).

101. Ding $Y$, Song Z, Liu Q et al. An enhanced chemotherapeutic effect facilitated by sonication of MSN. Dalton Trans. 46(35) 11875-11883 (2017).

MeHA hydrogel was incorporated with drug-loaded mesoporous silica nanoparticles (a sonosensitizer) to effectively kill target cancer cells through synergistic chemotherapy and sonodynamic therapy.

102. Chang $H$, Zheng $M, Y u X$ et al. A swellable microneedle patch to rapidly extract skin interstitial fluid for timely metabolic analysis. Adv. Mater. 29(37), (2017).

MeHA hydrogel was made into a microneedle patch to extract skin interstitial fluid for the sensing of metabolites, such as glucose and cholesterol, in a rapid and minimally invasive manner.

103.Gwon K, Kim E, Tae G. Heparin-hyaluronic acid hydrogel in support of cellula activities of 3D encapsulated adipose derived stem cells. Acta Biomater. 49 284-295 (2017).

104. Lin S, Lee WYW, Feng Q et al. Synergistic effects on mesenchymal stem cell-based cartilage regeneration by chondrogenic preconditioning and mechanical stimulation. Stem Cell Res. Ther. 8(1), 221 (2017).

105. Whitehead TJ, Avila COC, Sundararaghavan HG. Combining growth factor releasing microspheres within aligned nanofibers enhances neurite outgrowth. J. Biomed. Mater. Res., Part A 106(1), 17-25 (2018).

106.Poldervaart MT, Goversen B, De Ruijter $M$ et al. 3D bioprinting of methacrylated hyaluronic acid (MeHA) hydrogel with intrinsic osteogenicity. PloS One 12(6) 0177628 (2017)

07. Duan B, Yin Z, Kang LH, Magin RL, Butch- er JT. Active tissue stiffness modulation controls valve interstitial cell phenotype and osteogenic potential in 3D culture. Acta Biomater. 36, 42-54 (2016).

108. Yu J, Zhang Y, Sun W et al. Internalized compartments encapsulated nanogels for targeted drug delivery. Nanoscale 8(17) 9178-9184 (2016).

109.Zhou L, Chen E, Jin W, Wang Y, Zhou J, Wei S. Monomer zinc phthalocyanine/ upconversion nanoparticle coated with hyaluronic acid crosslinked gel as NIR light-activated drug for in vitro photodynamic therapy. Dalton Trans. 45(38) 15170-15179 (2016).

110.Wu S, Xu R, Duan B, Jiang P. Three-dimensional hyaluronic acid hydrogel-based models for in vitro human iPSC-derived NPC culture and differentiation. J. Mater Chem. B 5(21), 3870-3878 (2017).

111. Khoshakhlagh P, Moore MJ. Photoreactive interpenetrating network of hyaluronic acid and Puramatrix as a selectively tunable scaffold for neurite growth. Acta Biomater. 16, 23-34 (2015).

112. Hayami JW, Waldman SD, Amsden BG. Chondrocyte generation of cartilage-lik tissue following photoencapsulatio in methacrylated polysaccharide solution blends. Macromol. Biosci. 16(7), 1083-1095 (2016).

113. Beck EC, Barragan M, Libeer TB et at Chondroinduction from naturally derived cartilage matrix: a comparison between devitalized and decellularized cartilage encapsulated in hydrogel pastes. Tissu Eng., Part A 22(7-8), 665-679 (2016).

114.Duan B, Hockaday LA, Kapetanovic E, Kang KH, Butcher JT. Stiffness and adhesivity control aortic valve interstitia cell behavior within hyaluronic acid based hydrogels. Acta Biomater. 9(8), 7640-7650 (2013).

115.Duan B, Kapetanovic E, Hockaday LA Butcher JT. Three-dimensional printed trileaflet valve conduits using biological hydrogels and human valve interstitial cells. Acta Biomater. 10(5), 1836-1846 (2014).

116. Wang Z, Abdulla R, Parker B, Samanipour R, Ghosh S, Kim K. A simple and high-resolution stereolithography-based 3 D bioprinting system using visible light crosslinkable bioinks. Biofabrication 7(4) 045009 (2015)

117. Miri AK, Nieto D, Iglesias L et al. Microfluidics-enabled multimaterial maskless stereolithographic bioprinting. Adv Mater 30(27), 1800242 (2018).

118.Sirova M, Vlierberghe SV, Matyasova V et al. Immunocompatibility evaluation of hydrogel-coated polyimide implants for applications in regenerative medicine. J. Biomed. Mater. Res., Part A 102(6), 1982-1990 (2014).

119.Bryant SJ, Nuttelman CR, Anseth KS Cytocompatibility of UV and visible ligh photoinitiating systems on cultured $\mathrm{Nl}$ $\mathrm{H} / 3 \mathrm{~T} 3$ fibroblasts in vitro. J. Biomater. Sci. Polym. Ed. 11(5), 439-457 (2000).

120.Calderon G, Thai P Hsu C et al.

Tubulogenesis of co-cultured human iPS-derived endothelial cells and human mesenchymal stem cells in fibrin and gelatin methacrylate gels. Biomater. Sci. 5(8), 1652-1660 (2017)

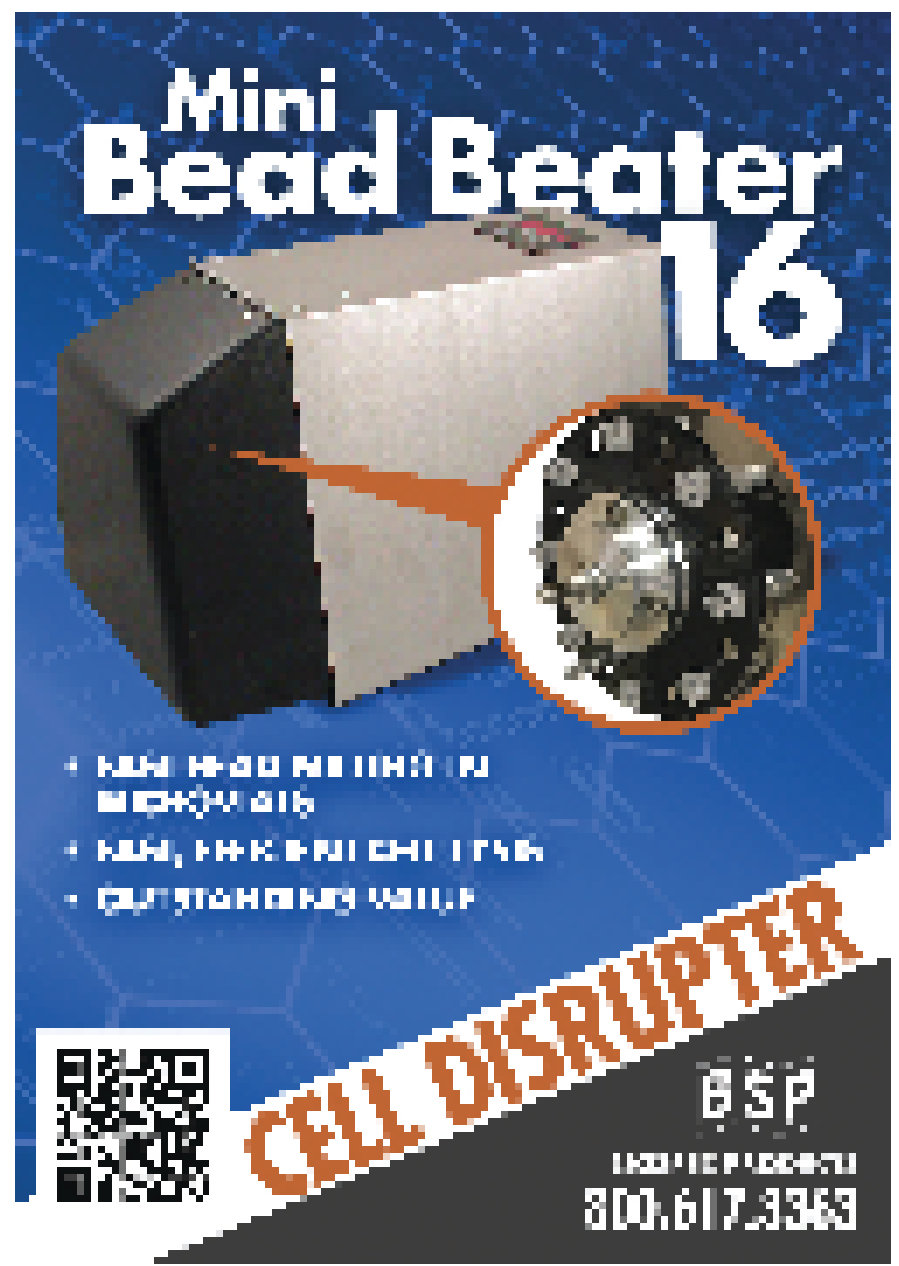

